NISSUNA UMANA INVESTIGAZIONE SI PUO DIMANDARE VERA SCIENZIA S'ESSA NON PASSA PER LE MATEMATICHE DIMOSTRAZIONI LEONARDO DA VINCI

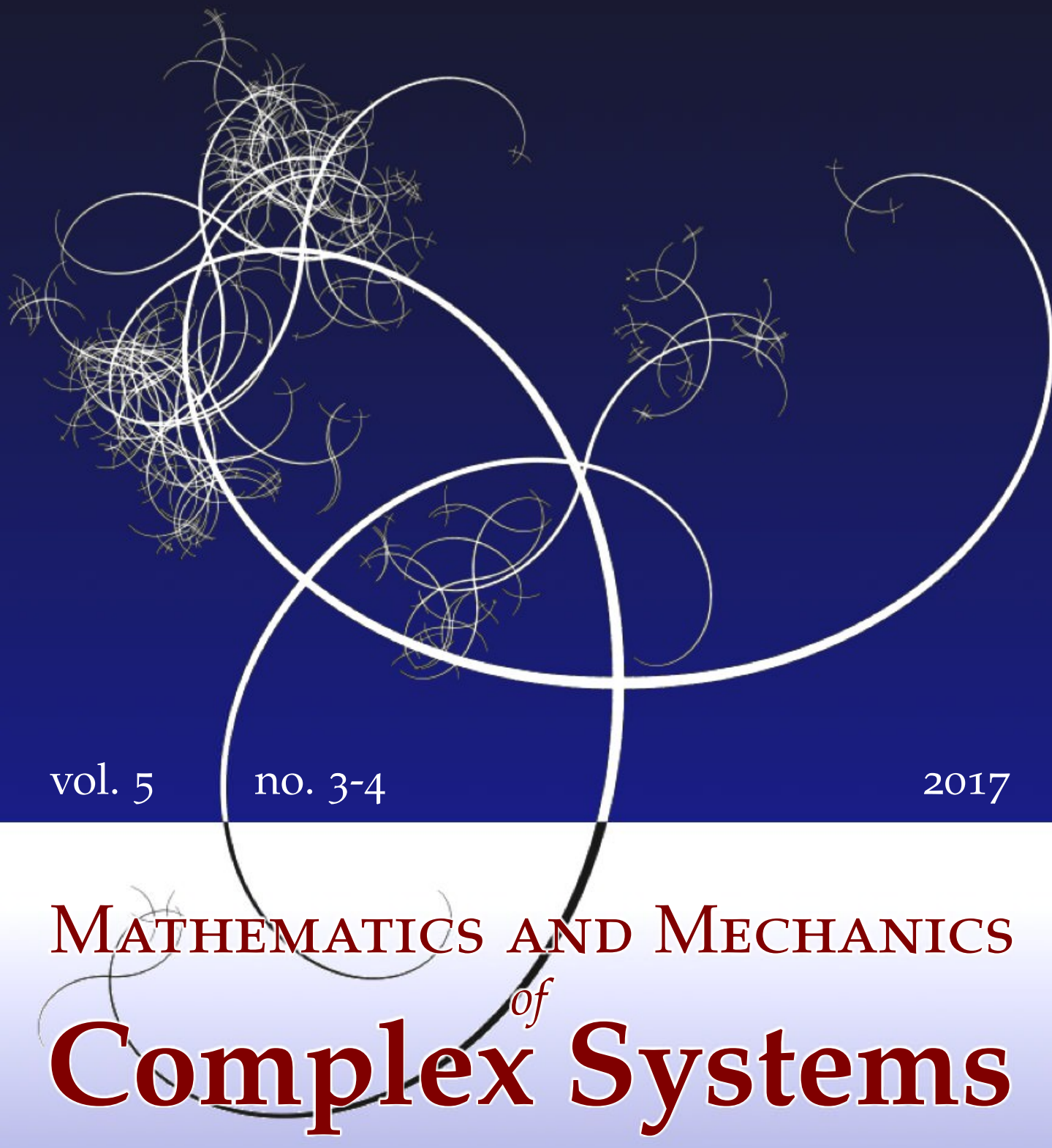

ROLF MAHNKEN

A VARIATIONAL FORMULATION FOR FUZZY ANALYSIS IN CONTINUUM MECHANICS 


\title{
A VARIATIONAL FORMULATION FOR FUZZY ANALYSIS IN CONTINUUM MECHANICS
}

\author{
ROLF MAHNKEN
}

\begin{abstract}
In order to improve the credibility of modern simulation tools, uncertainties of different kinds have to be considered. This work is focused on epistemic uncertainties in the framework of continuum mechanics, which are taken into account by fuzzy analysis. The underlying min-max optimization problem of the extension principle is approximated by $\alpha$-discretization, resulting in a separation of minimum and maximum problems. To become more universal, socalled quantities of interest are employed, which allow a general formulation for the target problem of interest. In this way, the relation to parameter identification problems based on least-squares functions is highlighted. The solutions of the related optimization problems with simple constraints are obtained with a gradient-based scheme, which is derived from a sensitvity analysis for the target problem by means of a variational formulation. Two numerical examples for the fuzzy analysis of material parameters are concerned with a necking problem at large strain elastoplasticity and a perforated strip at large strain hyperelasticity to demonstrate the versatility of the proposed variational formulation.
\end{abstract}

\section{Introduction}

The reliability assessment of components and engineering structures in today's industry is commonly performed by extensive use of numerical simulations. In order to improve its credibility uncertainties have to be taken into account, where two categories are distinguished, aleatoric and epistemic; see, e.g., [Sullivan 2015]. Aleatoric (or irreducible, stochastic) uncertainties refer to variability as a consequence of, e.g., fluctuations through time, variation across space, or manufacturing differences. In principle, they cannot be reduced by empirical effort; however, provided sufficient information is available they can be taken into account

\section{Communicated by Paul Steinmann.}

This work is based on investigations of SPP 1886: "Polymorphe Unschärfemodellierungen für den numerischen Entwurf von Strukturen", which is kindly supported by the Deutsche Forschungsgemeinschaft (DFG).

MSC2010: 74-XX.

Keywords: fuzzy analysis, $\alpha$-level optimization, quantities of interest, optimization with simple constraints, large strain elasticity, large strain elastoplasticity. 
with probabilistic concepts. Epistemic (reducible, subjective) uncertainties refer to subjectivity as a consequence of, e.g., incomplete scientific understanding or lack of measurements, which indicate a possible value range rather than a probability function. Contrary to aleatoric uncertainties, in principle they can be reduced by empirical effort, e.g., investing more in measurement.

Methodologies for the modeling of epistemic uncertainties are, e.g., interval analysis and, increasingly applied over the last few years, fuzzy analysis. Though the development of fuzzy arithmetic was started in the 1960s by Zadeh [1965], its application in solid mechanics is a rather new research area. Fuzzy analysis allows one to identify sensitivities of the response of a structure with respect to the magnitude of imprecision of the input data. The underlying axiomatic formalism is provided by the extension principle [Möller and Beer 2004]. It specifies the mapping of the fuzzy input set into a new fundamental set and results in solution of a maximum-minimum problem. In order to alleviate the complexity of this demanding task, $\alpha$-level optimization is employed. Here the membership input functions are discretized and maximum-minimum problems on each level eventually render a discretized solution for the membership output functions. The higher the chosen number of $\alpha$-levels, the greater the accuracy of the membership output of the response is.

Both the extension principle as well as $\alpha$-discretization may result in significant numerical efforts, in particular if the structural analysis is performed with the finite element method (FEM). A common methodology for fuzzy analysis with $\alpha$-discretization is vertex solution. Here, the extreme output responses to the FEMmodel for each $\alpha$-level are obtained by binary combinations of the fuzzy input variables; see, e.g., [Akpan et al. 2001]. Möller et al. [2000] employ a generic algorithm to perform the global optimization on every $\alpha$-level. Farkas et al. [2008] propose a parameter reduction scheme for the optimization problem. In order to accelerate optimization for the dynamic fuzzy FE analysis, De Munck et al. [2008] introduce a surrogate model, which replaces the real response of the analysis based on only a few computed values. Muhanna and Mullen [1999] employ the concept of $\alpha$-cuts within a fuzzy finite element method with applications for truss, beam, and plate problems in solid mechanics.

The above publications are typically formulated in terms of the underlying finite element discretized structure. To the author's knowledge, the variational formulation for fuzzy analysis of problems within the framework of continuum mechanics has not been considered so far. The present study intends to close this gap. Variational formulations have at least two intrinsic advantages:

- They enable a general framework for the analytical sensitivity analysis, which allows the effective solution of the related optimization problems with gradientbased methods; see, e.g., [Mahnken 2004]. 
- They give the basis for adaptive refinement of the underlying FE discretization [Rüter 2004; Johansson et al. 2007; Bangerth and Rannacher 2003; Widany and Mahnken 2012; Mahnken 2013].

Fuzzy analysis typically is performed for discrete values, e.g., the displacement at a certain finite element node. However, in some cases one might be interested in quantities within certain subregions of a structure. To this end, we introduce a so-called quantity of interest (or goal function) as an output quantity of the fuzzy analysis. Quantities of interest have been given much attention, e.g., in adaptive strategies over the last several years; see, e.g., [Rüter 2004; Johansson et al. 2007; Bangerth and Rannacher 2003; Widany and Mahnken 2012; Mahnken 2013].

This paper is structured as follows. Section 2 establishes the state problem and the target problem. Starting from basics of continuum mechanics, a weak formulation for the mechanical equilibrium is derived. Furthermore, we propose examples for a quantity of interest, which are used as output quantities for the fuzzy analysis. Section 3 incorporates the variational formulation into the optimization concepts of the extension principle and $\alpha$-level optimization. Section 4 provides the resulting optimality conditions in a variational setting. To this end, first- and second-order sensitivity analyses are performed by both the direct differentiation method as well as the adjoint state differentiation method. Moreover, we highlight the relation of least-squares problems to $\alpha$-level optimization. Section 5 briefly describes the finite element discretization followed by Section 6, which provides a brief overview on deterministic, gradient-based optimization methods. Representative examples in Section 7 demonstrate the versatility of the proposed variational formulation.

Notations. Symbolic tensor notation is employed mostly, if not stated otherwise. The scalar products of two vectors $\boldsymbol{a}$ and $\boldsymbol{b}$ as well as two second-order tensors $\boldsymbol{A}$ and $\boldsymbol{B}$ are, respectively,

$$
\boldsymbol{a} \cdot \boldsymbol{b}=a_{i} b_{i}, \quad \boldsymbol{A}: \boldsymbol{B}=A_{i j} B_{i j},
$$

using the Einstein summation rule for repeated indices $i, j=1,2,3$. The action of a second-order tensor $\boldsymbol{A}$ on a vector $\boldsymbol{b}$ is represented by $\boldsymbol{A} \cdot \boldsymbol{b}$. The tensor product $\boldsymbol{a} \otimes \boldsymbol{b}$ of two vectors $\boldsymbol{a}$ and $\boldsymbol{b}$ is a second-order tensor defined by the relation $(\boldsymbol{a} \otimes \boldsymbol{b}) \cdot \boldsymbol{b}=$ $(\boldsymbol{b} \cdot \boldsymbol{c}) \boldsymbol{a}$ for all vectors $\boldsymbol{c}$. 1 and $\square$ are the second- and fourth-order unit tensors, respectively, with the properties $\mathbf{1} \cdot \boldsymbol{c}=\boldsymbol{c}$ and $\square: \boldsymbol{C}=\boldsymbol{C}$ for all vectors $\boldsymbol{c}$ and all tensors $\boldsymbol{C}$. The nabla operator $\nabla$ with respect to initial coordinates $X$ is introduced, such that

$$
\nabla(\cdot)=\frac{\partial(\cdot)}{\partial X_{i}} \boldsymbol{e}_{i} \Rightarrow \nabla \boldsymbol{c} \equiv \boldsymbol{c} \otimes \nabla=c_{i, j} \boldsymbol{e}_{i} \otimes \boldsymbol{e}_{j} \quad \text { with } c_{i, j}=\frac{\partial c_{i}}{\partial X_{j}}
$$

where $\boldsymbol{e}_{i}, i=1,2,3$, is a Cartesian orthonormal basis and $X_{i}$ are coordinates of the reference placement $\boldsymbol{X}$. 


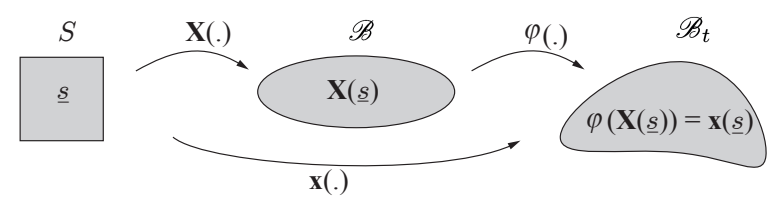

Figure 1. Mapping from design to configurations.

\section{State and target problem}

2.1. Strong and weak equilibrium problem. We consider a continuum body $\boldsymbol{B}$ occupying the reference (initial) configuration $\mathscr{B} \subset \mathscr{E}^{3}$ within the Euclidean space $\mathscr{E}^{3}$. Material particles are labeled by the placement vector $\boldsymbol{X} \in \mathscr{B}$. The surface of $\mathscr{B}$ is denoted as $\partial \mathscr{B}$, is assumed smooth, and has unit normal vector $N$. The time is denoted by $t \in \mathcal{T}$, where $\mathcal{T}=[0, T] \subset \mathbb{R}_{+}$is the time domain. Note that for time-independent problems the variable $t$ also provides a convenient history parameter in order to label the sequence of events and quasistatic conditions. In addition, we introduce $n_{s}$ design parameters $\left[s_{1}, \ldots, s_{n_{s}}\right]=\underline{s} \in \mathcal{S}$ within a design space $\mathcal{S}=\mathcal{S}_{1} \times \mathcal{S}_{2} \times \cdots \times \mathcal{S}_{n_{s}} \subset \mathbb{R}^{n_{s}}$. These may represent several influences on the structure, such as material, structural, or loading parameters, geometric properties or boundary conditions, etc. In this paper, the influence of uncertainty for the design parameters is taken into account by fuzzy analysis.

In order to relate design space $\mathcal{S}$ and time domain $\mathcal{T}$ as well as initial and current configurations $\mathscr{B}$ and $\mathscr{B}_{t}$, respectively, the following configurational mapping is introduced (see also [Barthold 1993] in the context of shape optimization):

$$
\boldsymbol{\varphi}(\cdot):=\left\{\begin{array}{c}
\mathscr{B} \times \mathcal{T} \times \mathcal{S} \rightarrow \mathscr{B}_{t} \subset \mathscr{E}^{3}, \\
\boldsymbol{X}(\underline{s}), t(\underline{s}), \underline{s} \mapsto \boldsymbol{x}(\underline{s})=\boldsymbol{\varphi}(\boldsymbol{X}(\underline{s}), t(\underline{s}), \underline{s}) .
\end{array}\right.
$$

The mappings $\boldsymbol{X}(\cdot): \mathcal{S} \rightarrow \mathscr{B} \subset \mathscr{E}^{3}$ and $t(\cdot): \mathcal{S} \rightarrow \mathcal{T} \subset \mathbb{R}_{+}$reflect dependencies of the initial geometry and the time, respectively, on design parameters. Here $\boldsymbol{x}(\cdot): \mathcal{S} \rightarrow \mathscr{B}_{t} \subset \mathscr{E}^{3}$ is a reduced placement operator, which relates the design space $\mathcal{S}$ and the actual configuration by means of the initial placement $\boldsymbol{X}$ and time $t$, such that $\boldsymbol{x} \in \mathscr{B}_{t}$ is the placement vector of a related particle at the current configuration $\mathscr{B}_{t} \subset \mathscr{E}^{3}$. The mappings $\boldsymbol{X}(\cdot), \boldsymbol{x}(\cdot)$, and $\varphi(\cdot)$ are visualized in Figure 1 , where for simplicity time dependence and explicit design dependence are not considered in the illustration.

Based on the configurational mapping in (3) we introduce three well known kinematical quantities of continuum mechanics

$$
\begin{aligned}
\boldsymbol{F} & =\nabla \boldsymbol{\varphi}(\boldsymbol{X}, t, \underline{s}), \\
J & =\operatorname{det} \boldsymbol{F}, \\
\boldsymbol{E} & =\frac{1}{2}\left(\boldsymbol{F}^{T} \cdot \boldsymbol{F}+\mathbf{1}\right),
\end{aligned}
$$


where $\boldsymbol{F}$ is the deformation gradient, which is known to be a mixed-variant, twofield tensor, $J$ is its determinant satisfying $J>0$, and $\boldsymbol{E}$ is the Green-Lagrange strain tensor.

Neglecting inertia terms, a strong formulation for the mechanical equilibrium problem is given as

$$
\begin{aligned}
& \text { Mechanical equilibrium } \quad \boldsymbol{\sigma} \cdot \nabla_{\boldsymbol{x}}+\overline{\boldsymbol{b}}(\underline{s})=\mathbf{0} \quad \text { in } \mathscr{B}_{t} \times \mathcal{S} \times \mathcal{T}, \\
& \text { Constitutive equations } \quad \sigma=\sigma(\varphi) \text { in } \mathscr{B}_{t} \times \mathcal{S} \times \mathcal{T}, \\
& \text { Boundary conditions } \quad \boldsymbol{u}=\overline{\boldsymbol{u}}(\underline{s}) \quad \text { on } \partial \mathscr{B}_{t u} \times \mathcal{S} \times \mathcal{T}, \\
& \boldsymbol{\sigma} \cdot \boldsymbol{n}=\overline{\boldsymbol{t}}(\underline{s}) \quad \text { on } \partial \mathscr{B}_{t \sigma} \times \mathcal{S} \times \mathcal{T} \text {. }
\end{aligned}
$$

Here, in addition to the previous notations we use $\sigma$ as the symmetric Cauchy stress tensor, $\nabla_{x}$ as the nabla operator with respect to the current placement vector $\boldsymbol{x}, \overline{\boldsymbol{b}}(\underline{s})$ as the body force vector per unit volume with respect to the current configuration, dependent on the (uncertain) design variables $\underline{s}$, and $\boldsymbol{u}=\boldsymbol{x}-\boldsymbol{X}$ as the displacement vector. The Dirichlet and Neumann boundary conditions (5c) and (5d), respectively, are formulated on the boundaries $\partial \mathscr{B}_{t u}$ and $\partial \mathscr{B}_{t \sigma}$, where the complementary conditions $\partial \mathscr{B}_{t u} \cup \partial \mathscr{B}_{t \sigma}=\partial \mathscr{B}_{t}$ and $\partial \mathscr{B}_{t u} \cap \partial \mathscr{B}_{t \sigma}=\varnothing$ hold, and where we defined traction forces $\overline{\boldsymbol{t}}(\underline{s})$ as well as prescribed displacements $\overline{\boldsymbol{u}}(\underline{s})$, both dependent on the (uncertain) design variables $\underline{s}$. In addition, $\boldsymbol{n}$ denotes a normal vector on the boundary $\partial \mathscr{B}_{t \sigma}$. A more general formulation for inelastic problems would require internal variables and their evolution equations, which however are left out here in order to alleviate the notation.

A weak formulation of the equilibrium problem is based on Hilbert spaces $\mathscr{U}$ for the displacement field $\boldsymbol{u} \in \mathscr{U}$ and $\mathscr{U}^{0} \subset \mathscr{U}$, which reflects zero Dirichlet boundary conditions for test functions $\delta \boldsymbol{u} \in \mathscr{U}^{0}$. Multiplying (5a) with test functions $\delta \boldsymbol{u} \in \mathscr{U}^{0}$, applying the divergence theorem, and incorporating the boundary conditions (5c)(5d), a weak formulation for the mechanical equilibrium is derived as

$$
\begin{aligned}
& \text { The state problem. For given } \underline{s} \in \mathcal{S} \text {, find } \boldsymbol{u}(\underline{s}) \in \mathscr{U} \text { such that } \\
& g(\underline{s}):=G(\underline{s}, \boldsymbol{u}(\underline{s}) ; \delta \boldsymbol{u}):=a(\underline{s}, \boldsymbol{u}(\underline{s}) ; \delta \boldsymbol{u})-\bar{l}(\delta \boldsymbol{u})=0 \text { for all } \delta \boldsymbol{u} \in \mathscr{U}^{0} .
\end{aligned}
$$

Here, the external and internal parts of the weak mechanical equilibrium equation are

$$
a(\boldsymbol{u}, \underline{s} ; \delta \boldsymbol{u})=\int_{\mathscr{B}_{t}} \boldsymbol{\sigma}: \boldsymbol{d}_{\delta} d v, \quad \bar{l}(\delta \boldsymbol{u})=\int_{\mathscr{B}_{t}} \overline{\boldsymbol{b}} \cdot \delta \boldsymbol{u} d v+\int_{\partial \mathscr{B}_{t \sigma}} \overline{\boldsymbol{t}} \cdot \delta \boldsymbol{u} d a,
$$

respectively, where $\boldsymbol{d}_{\delta}=\nabla_{x} \operatorname{sym}(\delta \boldsymbol{u})$ is a rate of deformation tensor, induced by the virtual displacement $\delta \boldsymbol{u} \in \mathscr{U}^{0}$; see Appendix B and Table B.1 for more details. Thus, $a(\cdot, \cdot ; \cdot)$ is a semilinear form on the Hilbert space $\mathcal{S} \times \mathscr{U} \times \mathscr{U}^{0}$, where linear and nonlinear dependent variables are separated by a semicolon. In the above, $\bar{l}(\cdot)$ 
is a linear functional with a dual pairing between the loading $\overline{\boldsymbol{b}}, \overline{\boldsymbol{t}} \in \mathscr{U}^{\prime}$ and $\boldsymbol{u} \in \mathscr{U}$, where $\mathscr{U}^{\prime}$ is the dual space of $\mathscr{U}$. The relation $G(\underline{s}, \boldsymbol{u}(\underline{s}) ; \delta \boldsymbol{u})=0$ is referred to as the state equation. We assume existence and uniqueness for the solution $\boldsymbol{u}(\underline{s})$ of the state problem (6), such that $g: \mathcal{S} \rightarrow \mathbb{R}$ defines a reduced solution operator.

For iterative solution of (6), with "frozen" design variables $\underline{s}$, we employ a Newton method at the current state $\tilde{\boldsymbol{u}}$ :

$$
\begin{aligned}
G_{\boldsymbol{u}}^{\prime}(\underline{s}, \tilde{\boldsymbol{u}}(\underline{s}) ; \delta \boldsymbol{u}, \Delta \boldsymbol{u}) & =-G(\underline{s}, \tilde{\boldsymbol{u}}(\underline{s}) ; \delta \boldsymbol{u}) \quad \text { for all } \delta \boldsymbol{u} \in \mathscr{U}^{0} \\
\Longrightarrow \boldsymbol{u} & =\tilde{\boldsymbol{u}}+\Delta \boldsymbol{u} .
\end{aligned}
$$

The generally unsymmetric tangent bilinear form $G_{\boldsymbol{u}}^{\prime}(\cdot, \cdot ; \cdot, \cdot)$ denotes the partial derivative with respect to $\boldsymbol{u}$. It is defined as a Gâteaux derivative at the point $\tilde{\boldsymbol{u}}$ in the direction of the virtual variation $\Delta \boldsymbol{u}$, i.e.,

$$
G_{\boldsymbol{u}}^{\prime}(\underline{s}, \tilde{\boldsymbol{u}} ; \delta \boldsymbol{u}, \Delta \boldsymbol{u}):=\lim _{\varepsilon \rightarrow 0} \varepsilon^{-1}(G(\underline{s}, \tilde{\boldsymbol{u}}+\varepsilon \Delta \boldsymbol{u} ; \delta \boldsymbol{u})-G(\underline{s}, \tilde{\boldsymbol{u}} ; \delta \boldsymbol{u})),
$$

assuming that the limit exists. The solution $\Delta \boldsymbol{u}$ of (8a) renders the new iterate $\boldsymbol{u}$ in (8b). More detailed formulations of the state equation $G_{\boldsymbol{u}}(\cdot, \cdot ; \cdot)$ and the tangent bilinear form $G_{\boldsymbol{u}}^{\prime}(\cdot, \cdot ; \cdot, \cdot)$ are provided in Appendix B.

2.2. Quantity of interest and target problem. In the subsequent exposition we do not merely want to characterize the fuzzy properties of the displacement field $\boldsymbol{u}(\underline{s})$ satisfying the state problem (6). Instead we are more interested in the fuzzy analysis of a physical event or a feature of the structure that depends upon $\boldsymbol{u}$. Such quantities of interest are characterized by functionals $Q(\boldsymbol{u})$ of the solutions $\boldsymbol{u}$ to (6). Quantities of interest have been given much attention in adaptive strategies over the last several years; see, e.g., [Rüter 2004; Johansson et al. 2007; Bangerth and Rannacher 2003; Widany and Mahnken 2012; Mahnken 2013] and, in the context of multiscale modeling, [Oden et al. 2006]. Possible realizations of the quantity of interest may be

(1) displacement at a certain point of the body $P \in \boldsymbol{B}$ with coordinates $\boldsymbol{X}_{P} \in \mathscr{B}$ at the end of loading with time $t=T$

$$
Q_{X}(\boldsymbol{u})=\left\|\boldsymbol{u}\left(\boldsymbol{X}_{P}, T\right)\right\|_{\mathcal{D}},
$$

(2) displacements at certain regions $\mathscr{B}_{m} \subset \mathscr{B}$ and certain time intervals $\mathcal{T}_{n} \subset \mathcal{T}$

$$
Q_{u}(\boldsymbol{u})=\int_{\mathcal{T}_{n}} \int_{\mathscr{B}_{m}}\|\boldsymbol{u}\|_{\mathcal{D}} d V d t
$$

(3) strains, e.g., of Green-Lagrange type according to (4), at certain regions $\mathscr{B}_{m} \subset$ $\mathscr{B}$ and certain time intervals $\mathcal{T}_{n} \subset \mathcal{T}$

$$
Q_{E}(\boldsymbol{u})=\int_{\mathcal{T}_{n}} \int_{\mathscr{B}_{m}}\|\boldsymbol{E}(\boldsymbol{u})\|_{\mathcal{D}} d V d t
$$


(4) stresses at certain regions $\mathscr{B}_{m} \subset \mathscr{B}$ and certain time intervals $\mathcal{T}_{n} \subset \mathcal{T}$

$$
Q_{P}(\boldsymbol{u})=\int_{\mathcal{T}_{n}} \int_{\mathscr{B}_{m}}\|\boldsymbol{P}(\boldsymbol{u})\|_{\mathcal{D}} d V d t
$$

and where $\boldsymbol{P}=J \boldsymbol{\sigma} \cdot \boldsymbol{F}^{-T}$ is the first Piola-Kirchhoff stress tensor,

(5) stresses at the boundary of prescribed displacements $\partial \mathscr{B}_{u} \subset \partial \mathscr{B}$ and time intervals $\mathcal{T}_{n} \subset \mathcal{T}$

$$
Q_{F}(\boldsymbol{u})=\int_{\mathcal{T}_{n}} \int_{\partial \mathscr{B}_{u}}\left\|\boldsymbol{P}(\boldsymbol{u})^{T} \cdot \boldsymbol{N}\right\|_{\mathcal{D}} d A d t
$$

such that the goal function has the interpretation of (a sum of) reaction forces,

(6) functional dependencies of design variables

$$
Q_{s}=Q(\underline{s})
$$

(7) or a combination of the above quantities with adequate weighting.

The standard choice of norm $\|\cdot\|_{\mathcal{D}}$ in the above definitions is a weighted $L^{2}$-norm.

Moreover, a comparison of the state variable $\boldsymbol{u}(\underline{s}) \in \mathscr{U}$ with experimental data $\boldsymbol{u}^{*} \in \mathscr{U}^{*}$ could be integrated in the quantity of interest, where $\mathscr{U}^{*}$ is the space of true data, that is, without measurement errors. To this end, we define the mappings

$$
\begin{aligned}
& \underline{\bar{d}}=\underline{\bar{D}}\left(\boldsymbol{u}^{*}\right) \quad \in \mathcal{D} \subset \mathbb{R}^{n_{d}}, \\
& \underline{d}(\underline{s}):=\underline{D}(\boldsymbol{u}(\underline{s})) \in \mathcal{D} \subset \mathbb{R}^{n_{d}} \text {. }
\end{aligned}
$$

Equation (15a) introduces an observation operator $\underline{\bar{D}}: \mathscr{U}^{*} \rightarrow \mathcal{D}$ mapping the trajectory of true data $\boldsymbol{u}^{*}$ to points $\underline{\bar{d}}=\underline{\bar{D}}\left(\boldsymbol{u}^{*}\right)$ in the data space $\mathcal{D} \in \mathbb{R}^{n_{d}}$ [Banks and Kunisch 1989]. Equation (15b) introduces a second observation operator $\underline{D}: \mathscr{U} \rightarrow \mathcal{D}$, which maps the state variable $\boldsymbol{u}(\underline{s}) \in \mathscr{U}$ to points $\underline{D}(\boldsymbol{u}(\underline{s}))$ in the data space $\mathcal{D} \in \mathbb{R}^{n_{d}}$. Moreover, in order to point out the "design driven" data as a result of the state problem (6), (15b) introduces the reduced observation operator $\underline{d}: \mathcal{S} \rightarrow \mathcal{D}$ to transform design variables $\underline{s}$ to the observation space [Vexler 2004]. In index notation (15b) can be written as $d_{k}(\underline{s}):=D_{k}(\boldsymbol{u}(\underline{s})), k=1, \ldots, n_{d}$. Hereafter, we assume $n_{d} \geq n_{s}$ for the number of experimental data $n_{d}$ and for the number of design variables $n_{s}$ (in this case material parameters), and we assume both operators $\underline{D}$ and $\underline{D}$ to be linear. With these notations a model error with respect to the data space $\mathcal{D} \subset \mathbb{R}^{n_{d}}$ becomes

$$
\underline{e}_{\mathrm{Mod}}=\underline{D}(\boldsymbol{u}(\underline{s}))-\underline{\bar{D}}\left(\boldsymbol{u}^{*}\right)=\underline{d}(\underline{s})-\underline{\bar{d}} \in \mathcal{D} \subset \mathbb{R}^{n_{d}}
$$


as the difference of simulated and experimental data. Using a weighted $L^{2}$-norm a quantity of interest may become

$$
\begin{array}{r}
Q_{\mathrm{LS}}(\boldsymbol{u}(\underline{s}))=\frac{1}{2} \int_{\mathcal{T}_{n}} \int_{\mathscr{B}_{m}}\left\|\underline{D}(\boldsymbol{u}(\underline{s}))-\underline{\bar{D}}\left(\boldsymbol{u}^{*}\right)\right\|_{\mathcal{D}}^{2} d V d t=\frac{1}{2}\|\underline{W}(\underline{d}(\underline{s}))-\underline{\bar{d}}\|_{2}^{2} \\
=: q_{\mathrm{LS}}(\underline{s}) .
\end{array}
$$

In the third part of (17) we have introduced a weighting matrix $\underline{W} \in \mathbb{R}^{n_{d} \times n_{d}}$, which accounts, e.g., for different physical dimensions or reasonable scaling for the quantities of interest on the region $\mathscr{B}_{m} \subset \mathscr{B}$ and the time interval $\mathcal{T}_{n} \subset \mathcal{T}$. Analogously to the reduced solution operator $g: \mathcal{S} \rightarrow \mathbb{R}$ in (6), $q_{\mathrm{LS}}: \mathcal{S} \rightarrow \mathbb{R}$ in (17) represents a reduced quantity of interest operator. Of course, the quantity of interest in (17) is nothing else but the least-squares functional in identification problems. Here, the interest is to minimize the distance between simulated data and experimental data in a least-squares sense; see, e.g., [Mahnken 2004]. Upon neglecting measurement errors the functional in (17) also has the interpretation of a mean for the model error in (16).

With a specific choice for the quantity of interest $Q$ at hand, for example according to (10)-(14) or (17), we formulate:

The target problem. For given $\underline{s} \in \mathcal{S}$, find $z:=q(\underline{s}):=Q(\underline{s}, \boldsymbol{u}(\underline{s}))$.

Note that due to the dependency $\boldsymbol{u}(\underline{s})$, solution of the target problem (18) involves solution of the state problem (6). In the following exposition, we assume a continuous mapping for the reduced quantity of interest operator $q: \mathcal{S} \rightarrow \mathbb{R}$ between the design variables $\underline{s}$ and the quantity of interest $q$ and a unique solution $\boldsymbol{u}(\underline{s})$.

\section{Fuzzy analysis}

3.1. Fuzzy set and fuzzy number. So far, the design space $\mathcal{S} \subset \mathbb{R}^{n_{s}}$ in the previous section has been assumed a precise set or a fundamental set [Möller and Beer 2004] of ordered pairs

$$
\begin{aligned}
\mathcal{S} & =\left\{\left(\underline{s}, \underline{\xi}_{\mathcal{S}}(\underline{s})\right) \mid \underline{s} \in \mathbb{R}^{n_{s}}, \underline{\xi}_{S}(\underline{s})=\{0,1\}^{n_{s}}\right\} \\
& =\mathcal{S}_{1} \times \mathcal{S}_{2} \times \cdots \times \mathcal{S}_{n_{s}} \subset \mathbb{R}^{2 n_{s}}, \\
\text { where } \quad \mathcal{S}_{i} & =\left\{\left(s_{i}, \xi_{\mathcal{S} i}\left(s_{i}\right)\right) \mid s_{i} \in \mathbb{R}, \xi_{\mathcal{S i}}\left(s_{i}\right) \in\{0,1\}\right\} \subset \mathbb{R}^{2}, \\
\xi_{\mathcal{S} i} & : \mathbb{R} \rightarrow\{0,1\}, \quad s_{i} \mapsto \begin{cases}1 & \text { if } s_{i} \in \mathcal{S}_{i}, \\
0 & \text { if } s_{i} \notin \mathcal{S}_{i} .\end{cases}
\end{aligned}
$$

According to (19b) the Cartesian product $\mathcal{S}$ comprises all combinations of the design variables $s_{1}, \ldots, s_{n_{s}}$ of the precise sets $\mathcal{S}_{i}$ in (19c). The corresponding indicator functions $\xi_{\mathcal{S} i}$ (19d) specify crisp interval boundaries for each design variable $s_{i}, i=1, \ldots, n_{s}$, in a precise manner; see, e.g., [Viertl 1996]. However, due to 

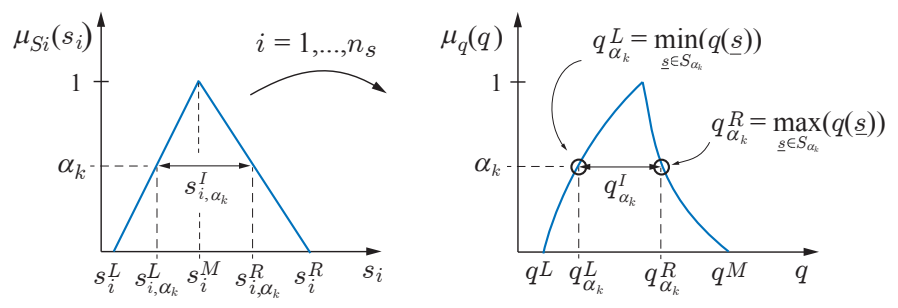

Figure 2. Schematic graph of fuzzy analysis with $\alpha$-level optimization. Left: input membership function, fuzzy triangular number $\hat{A}_{i}=\left\langle s_{i}^{L}, s_{i}^{M}, s_{i}^{R}\right\rangle$, and $\alpha$-levels. Right: output membership function.

imprecision of measurements this is not realistic. Therefore, in fuzzy analysis, the precise set $\mathcal{S}$ is replaced by a nonprecise set, or fuzzy set [Möller and Beer 2004] of ordered pairs

$$
\begin{aligned}
\hat{\mathcal{S}} & =\left\{\left(\underline{s}, \mu_{S}(\underline{s})\right) \mid \underline{s} \in \mathbb{R}^{n_{s}}, \mu_{S}(\underline{s})=\min _{i=1, \ldots, n_{s}}\left\{\mu_{\mathcal{S} i}\left(\underline{s}_{i}\right)\right\}\right\} \\
& =\hat{\mathcal{S}}_{1} \times \hat{\mathcal{S}}_{2} \times \cdots \times \hat{\mathcal{S}}_{n_{s}} \subset \mathbb{R}^{2 n_{s}}, \\
\hat{\mathcal{S}}_{i} & =\left\{\left(s_{i}, \mu_{\mathcal{S} i}\left(s_{i}\right)\right) \mid s_{i} \in \mathbb{R}, \mu_{\mathcal{S} i}\left(s_{i}\right) \in[0,1]\right\} \subset \mathbb{R}^{2}, \\
\mu_{\mathcal{S} i} & : \mathbb{R} \rightarrow[0,1], \quad s_{i} \mapsto 0 \leq \mu_{\mathcal{S} i}\left(\underline{s}_{i}\right) \leq 1 .
\end{aligned}
$$

According to (20a) and (20b) the Cartesian product $\hat{\mathcal{S}}$ comprises all combinations of the design variables $s_{1}, \ldots, s_{n_{s}}$. Equation (20c) is the one-dimensional fuzzy set where in (20d), for each set $\hat{\mathcal{S}}_{i}$ the indicator function $\xi_{\mathcal{S} i}\left(s_{i}\right)$ in $(19 \mathrm{~d})$ is replaced by a membership function $\mu_{\mathcal{S} i}\left(s_{i}\right)$, which represents a gradual weighting of each design space $\mathcal{S}_{i}$. Subsequently, only normalized membership functions with the property $\sup _{s_{i} \in \mathcal{S}_{i}}\left[\mu_{\mathcal{S} i}\left(s_{i}\right)\right]=1$ are considered. The closer $\mu_{\mathcal{S} i}\left(s_{i}\right)$ is to 1 , the more the element $s_{i}$ belongs to the set $\hat{\mathcal{S}}_{i}$; the closer $\mu_{\mathcal{S} i}\left(s_{i}\right)$ is to 0 , the less the element $s_{i}$ belongs to the set $\hat{\mathcal{S}}_{i}$. A convex and normalized fuzzy set is referred to as a fuzzy number. Figure 2, left, illustrates a triangular fuzzy number

$$
\hat{A}_{i}=\left\langle s_{i}^{L}, s_{i}^{M}, s_{i}^{R}\right\rangle, \quad \text { where } s_{i}^{L}<s_{i}^{M}<s_{i}^{R},
$$

with in-between value $s_{i}^{M}$ and lower and upper bounds $s_{i}^{L}, s_{i}^{R}$.

By definition of the reduced quantity of interest operator $q: \mathcal{S} \rightarrow \mathbb{R}$ in (17), the quantities of interest $Q$ in (10)-(14) and (17) for displacements, stresses, strains, etc., become functions of the design variables $s_{i}$ and consequently can be treated as fuzzy functions. The intrinsic goal of fuzzy analysis is to propagate the uncertainties of the fuzzy input variables, in our case the design variables $s_{i}, i=1, \ldots, n_{s}$, to a fuzzy output variable, in our case the quantity of interest $q(\underline{s})$ in the target problem (18). 
3.2. The extension principle. In order to perform mathematical operations with fuzzy numbers Zadeh developed the extension principle [Dubois and Prade 1980; Zadeh 1965; Möller and Beer 2004]. It specifies the mapping of the fuzzy input set $\hat{\mathcal{S}}$ into a new fundamental set $\mathcal{Z}$ with the aid of the mapping $z=q(\underline{s})$ defined in the target problem (18). On the fundamental set $\mathcal{Z}$ the fuzzy set $\tilde{\mathcal{B}}$ together with its membership function $\mu_{B}(z)$ is gained. The mapping $q(\underline{s})$ leads to the fuzzy set $\tilde{\mathcal{B}}$ on $\mathcal{Z}$ :

$$
\tilde{\mathcal{B}}=\left\{\left(z, \mu_{B}(z)\right) \mid z=q(\underline{s}), z \in \mathcal{Z} ; \underline{s} \in \mathcal{S}\right\},
$$

where

$$
\mu_{B}(z)= \begin{cases}\sup _{z=q(\underline{s})} \min \left(\mu_{i}\left(s_{1}\right), \ldots, \mu_{n_{s}}\left(s_{n_{s}}\right)\right) & \text { if there exists } z=q(\underline{s}), \\ 0 & \text { otherwise. }\end{cases}
$$

Here the membership values $\mu_{B}(z)$ are computed according to the sup-min problem (22b) in order to minimize and maximize the quantity of interest $q(\underline{s})$ defined in (18). Following Akpan et al. [2001] problem (22) can be interpreted as follows. For a crisp value of the output $z$, there exist zero, one, or more combinations of crisp values of input variables $\underline{s}$ such that $z=q(\underline{s})$. The possibility of each combination is given by the smallest possibility value for all fuzzy input variables. The possibility that $z=q(\underline{s})$ is given by the maximum possibility value for all these combinations.

As pointed out by Wasfy and Noor [1998] the direct solution of (22b) is intractable except for very simple fuzzy finite element problems involving at most one or two fuzzy variables. For a general function $q$, the number of operations needed to solve the state problem (6) is of the order $\infty^{n_{s}}$ [Wasfy and Noor 1998].

3.3. $\alpha$-level optimization. Due to its mathematical complexity and the significant numerical effort the extension principle, (22) can be approximated by the so-called $\alpha$-level optimization method. Here, as schematically illustrated in Figure 2, left, each membership input function $\mu_{i}$ is discretized into $n_{\alpha} \alpha$-cuts, where $0 \leq \alpha_{k} \leq 1$ denotes the membership level [Möller and Beer 2004]. For this purpose we define the crisp set

$$
\mathcal{S}_{i, \alpha_{k}}=\left\{s_{i} \mid \mu_{\mathcal{S} i, \alpha_{k}}\left(s_{i}\right) \geq \alpha_{k}, s_{i} \in \mathcal{S}_{i}\right\}=\left[s_{i, \alpha_{k}}^{L}, s_{i, \alpha_{k}}^{R}\right]:=s_{i, \alpha_{k}}^{I}, \quad i=1, \ldots, n_{s},
$$

of the fuzzy variable $s_{i}$ at level $\alpha_{k}$. It has crisp lower and upper bounds $s_{i, \alpha_{k}}^{L}$ and $s_{i, \alpha_{k}}^{R}$, respectively, and therefore defines a crisp interval as illustrated in Figure 2, left. Note also that the $\alpha$-cut $\mathcal{S}_{i, 0}=\left[s_{i}^{L}, s_{i}^{R}\right]$ is the support of the fuzzy set $\hat{\mathcal{S}}_{i}$. The intervals in (23) give rise to the definition of an $n_{s}$-dimensional constrained design space

$$
\mathcal{S}_{\alpha_{k}}=\left[\mathcal{S}_{1, \alpha_{k}} \times \cdots \times \mathcal{S}_{i, \alpha_{k}} \times \cdots \times \mathcal{S}_{n_{s}, \alpha_{k}}\right] \subset \mathcal{S} \subset \mathbb{R}^{n_{s}} .
$$


Then, the fuzzy response for each selected $\alpha_{k}$-level is obtained from the minimum and maximum values of the quantity of interest $q$ in (18) as

$$
\begin{aligned}
& \text { P1: } \alpha \text {-level optimization problems. } \\
& \text { Find } q_{\alpha_{k}}^{L}=\min _{\underline{s} \in \mathcal{S}_{\alpha_{k}}} q(\underline{s})=\min _{\underline{s} \in \mathcal{S}_{\alpha_{k}}} Q(\underline{s}, \boldsymbol{u}(\underline{s})) . \\
& \text { Find } q_{\alpha_{k}}^{R}=\max _{\underline{s} \in \mathcal{S}_{\alpha_{k}}} q(\underline{s})=\max _{\underline{s} \in \mathcal{S}_{\alpha_{k}}} Q(\underline{s}, \boldsymbol{u}(\underline{s})) .
\end{aligned}
$$

In this way, fuzzy analysis reduces to an interval-based analysis with crisp bounds. As visualized in Figure 2, right, the two extrema $q_{\alpha_{k}}^{L}$ and $q_{\alpha_{k}}^{R}$ render two points of the membership function $\mu_{q}(q)$ for the membership level $\alpha_{k}$. As, by assumption in Section 2.1, there is a continuous mapping $\boldsymbol{u}=\boldsymbol{u}(\underline{s})$ between the design variables $\underline{s}$ and the quantity of interest $q$ with a unique solution of the state problem (6), the interval

$$
q_{\alpha_{k}}^{I}=\left[q_{\alpha_{k}}^{L}, q_{\alpha_{k}}^{R}\right]
$$

is fully described by the lower and upper bounds $q_{\alpha_{k}}^{L}$ and $q_{\alpha_{k}}^{R}$. In addition we remark that due to the definition for $\mathcal{S}_{\alpha_{k}} \subset \mathbb{R}^{n_{s}}$ in (24) both optimization problems in (25) involve only simple constraints. Problems of this kind are treated extensively in [Bertsekas 1982].

\section{Optimality conditions for the $\alpha$-level optimization problems}

4.1. Preliminaries. The optimization problems (25a) and (25b) differ only in the specification of a minimum and a maximum. Therefore, without loss of generality following this section we will mainly resort to the minimum problem (25a), if not stated otherwise. At the same time, for notational brevity the upper index $L$ and the lower index $\alpha_{k}$ are left out. We also note that the present article is focused on the case of simple constraints in (23). This means that no interaction of the fuzzy variables as in the quantity of interest in (14) is considered, which will be a further challenge in future work on fuzzy analysis; see, e.g., [Möller and Beer 2004].

First-order necessary and second-order sufficient optimality conditions for a stable stationary point $\underline{s} \in \mathcal{S}_{\alpha_{k}}$ of the minimization problem (25a) are

$$
\begin{aligned}
q^{\prime}(\underline{s} ; \delta \underline{s}) & \geq 0, \\
q^{\prime \prime}(\underline{s} ; \delta \underline{s}, \delta \underline{s}) & \geq \gamma \| \delta \underline{s}_{\mathcal{S}_{\alpha_{k}}}^{2} \quad \text { for all } \delta \underline{s} \in \overline{\mathcal{S}}_{\alpha_{k}},
\end{aligned}
$$

where

$$
\overline{\mathcal{S}}_{\alpha_{k}}(\underline{s})=\left\{\delta \underline{s} \in \mathcal{S} \mid \text { there exists } \bar{\beta}>0 \text { such that } \underline{s}+\beta \delta \underline{s} \in \mathcal{S}_{\alpha_{k}}, 0 \leq \beta<\bar{\beta}\right\}
$$


is the space of admissible directions. Furthermore, in (27) we have $\gamma \in \mathbb{R}_{+}$; see, e.g., [Nocedal and Wright 1999]. For computing $q^{\prime}(\underline{s} ; \delta \underline{s})$ and $q^{\prime \prime}(\underline{s} ; \delta \underline{s})$ two conceptions are distinguished: the (more conventional) direct differentiation method and the (more formal) adjoint state method.

4.2. The direct differentiation method. Application of the chain rule to the reduced functionals in (6) and (25a) gives us

$$
\begin{array}{ll}
g^{\prime}(\underline{s} ; \delta \underline{s})=G_{\underline{s}}^{\prime}(\underline{s}, \boldsymbol{u}(\underline{s}) ; \delta \boldsymbol{u}, \delta \underline{s})+G_{\boldsymbol{u}}^{\prime}(\underline{s}, \boldsymbol{u}(\underline{s}) ; \delta \boldsymbol{u}, \bar{\delta} \boldsymbol{u})=0 & \text { for all } \delta \boldsymbol{u} \in \mathscr{U}^{0} \\
& \text { and } \delta \underline{s} \in \mathcal{S}_{\alpha_{k}} \\
q^{\prime}(\underline{s} ; \delta \underline{s})=Q_{\underline{s}}^{\prime}(\underline{s}, \boldsymbol{u}(\underline{s}) ; \delta \underline{s})+Q_{\boldsymbol{u}}^{\prime}(\underline{s}, \boldsymbol{u}(\underline{s}) ; \bar{\delta} \boldsymbol{u}) \quad \geq 0 & \text { for all } \delta \underline{s} \in \mathcal{S}_{\alpha_{k}}
\end{array}
$$

where $G_{s}^{\prime}(\cdot, \cdot ; \cdot), G_{\boldsymbol{u}}^{\prime}(\cdot, \cdot ; \cdot, \cdot)$, etc., denote partial derivatives with respect to $\underline{\underline{s}}$ and $\boldsymbol{u}$, defined as Gâteaux derivatives. The tangent problem in (29a) is zero since $\boldsymbol{u}(\underline{s})$ solves the state problem (6) for each $\underline{s} \in \mathcal{S}$, where $\bar{\delta} \boldsymbol{u}:=\boldsymbol{u}_{s}^{\prime}(\underline{s} ; \delta \underline{s})$ is the directional sensitivity along $\delta s$ [Johansson et al. 2007]. The inequality (29b) follows from the first-order optimality condition (27a). Inserting the solution $\bar{\delta} \boldsymbol{u}$ of the tangent problem (29a) into (29b) may be used to evaluate $q^{\prime}(\underline{s} ; \delta \underline{s})$. With this result for $q^{\prime}$ we introduce the gradient of the quantity of interest

$$
\nabla q(\underline{s}) \in \mathcal{S}, \quad \text { where }(\nabla q(\underline{s}))_{i}=q^{\prime}\left(\underline{s} ; \underline{e}_{i}\right), i=1, \ldots, n_{s},
$$

and where $\left(\underline{e}_{i}\right)_{j}=\delta_{i j}$ with the standard Kronecker delta. Then, for the case of simple constraints in (24), the necessary conditions (27a) and (29b) simplify as [Bertsekas 1982]

$$
\{\nabla q(\underline{s})\}_{i}=\frac{\partial q}{\partial s_{i}} \begin{cases}=0 & \text { if } s_{i, \alpha_{k}}^{L}<s_{i}<s_{i, \alpha_{k}}^{R} \\ \geq 0 & \text { if } s_{i}=s_{i, \alpha_{k}}^{L}, \\ \leq 0 & \text { if } s_{i}=s_{i, \alpha_{k}}^{R}\end{cases}
$$

A detailed study of the second-order optimality condition (27b) by means of the direct differentiation method is performed in Appendix A.1.

As an example, we consider the quantity of interest of least-squares type in (17). Exploiting $Q_{\underline{s}}^{\prime}(\underline{s}, \boldsymbol{u}(\underline{s}) ; \delta \underline{s})=0$ in $(29 \mathrm{~b})$, the gradient is derived from (29b) as

$$
\begin{aligned}
\nabla q_{\mathrm{LS}}(\underline{s}) & =(\underline{d}(\underline{s})-\underline{\bar{d}})^{T} \underline{W}^{T} \underline{W} \underline{J}, \\
\text { where } \underline{J} & =\nabla \underline{d}(\underline{s})=\underline{D}_{\boldsymbol{u}}^{\prime}(\boldsymbol{u}) \cdot \nabla \boldsymbol{u}(\underline{s}) .
\end{aligned}
$$

In (32b), the Jacobian matrix $\underline{J}$ is obtained by the chain rule applied to the reduced observation operator in (15) with relations $(\nabla \boldsymbol{u}(\underline{s}))_{i}=\boldsymbol{u}_{s}^{\prime}\left(\underline{s} ; \underline{e}_{i}\right)$. Moreover, exploiting $Q_{\underline{s} \underline{s}}^{\prime \prime}\left(\underline{s}, \boldsymbol{u}(\underline{s}) ; \delta \underline{s}_{1}, \delta \underline{s}_{2}\right)=Q_{\underline{s} \boldsymbol{u}}^{\prime \prime}\left(\underline{s}, \boldsymbol{u}(\underline{s}) ; \delta \underline{s}_{1}, \bar{\delta} \boldsymbol{u}_{2}\right)=\bar{Q}_{\underline{u} \underline{\underline{s}}}^{\prime \prime}\left(\underline{s}, \boldsymbol{u}(\underline{s}) ; \bar{\delta} \boldsymbol{u}_{1}, \bar{\delta} \boldsymbol{u}_{2}\right)=0$ in (A-1b), the Hessian of the reduced cost functional $q_{\mathrm{LS}}$ in (25a) is derived from 
$(\mathrm{A}-1 \mathrm{~b})$ as

$$
\begin{aligned}
& \nabla^{2} q_{\mathrm{LS}}(\underline{s})=\underline{J}^{T} \underline{W}^{T} \underline{W} \underline{J}+\underline{M} \\
& \text { where } \underline{M}=\sum_{i, j, k=1}^{n_{d}} W_{i j}\left(d_{j}(\underline{s})-\bar{d}_{j}\right) W_{i k}\left(D_{k}\right)_{\boldsymbol{u}}^{\prime}(\boldsymbol{u}) \cdot \nabla^{2} \boldsymbol{u}(\underline{s})
\end{aligned}
$$

with relations $\left(\nabla^{2} \boldsymbol{u}(\underline{s})\right)_{i j}=\boldsymbol{u}_{s S}^{\prime \prime}\left(\underline{s} ; \underline{e}_{i}, \underline{e}_{j}\right)$.

4.3. The adjoint state differentiation method. Let us consider the optimization problems

$$
\begin{aligned}
& \text { P2: } \alpha \text {-level optimization problems. } \\
& \text { (1) Find } Q_{\alpha_{k}}^{L}=\min _{\underline{s} \in \mathcal{S}_{\alpha_{k}}, \boldsymbol{u} \in \mathscr{U}} Q(\underline{s}, \boldsymbol{u}) \text {. (2) Find } Q_{\alpha_{k}}^{R}=\max _{\underline{\underline{S}} \in \mathcal{S}_{\alpha_{k}}, \boldsymbol{u} \in \mathscr{U}} Q(\underline{s}, \boldsymbol{u}) . \\
& G(\underline{s}, \boldsymbol{u} ; \delta \boldsymbol{u})=0 \text { for all } \delta \boldsymbol{u} \in \mathscr{U}^{0} . \\
& g_{j}=s_{j, \alpha_{k}}^{L}-s_{j} \leq 0, j=1, \ldots, n_{s} . \\
& g_{n_{s}+j}=s_{j}-s_{j, \alpha_{k}}^{R} \leq 0, j=1, \ldots, n_{s} .
\end{aligned}
$$

in order to minimize and maximize the quantity of interest $Q$. Equation (34b) constitutes the state problem (6) as an equality condition, and the inequalities (34c)-(34d) specify constraint functions associated to the $n_{s}$-dimensional space $\mathcal{S}_{\alpha_{k}}$ in (24). Contrary to problem P1 in (25), the design variables $\underline{s}$ and the displacement field $\boldsymbol{u}$ are independent variables. Consequently, there is no functional dependence $\boldsymbol{u}(\underline{s})$, and so far no use is made of the reduced quantity of interest operator $q: \mathcal{S} \rightarrow \mathbb{R}$ in the target problem (18) and the reduced solution operator $g: \mathcal{S} \rightarrow \mathbb{R}$ in the state problem (6). As in Section 4, without loss of generality, from now on we will resort only to the minimum problem with quantity of interest in (34a)(1). If there is no risk of confusion, the lower index $\alpha_{k}$ is left out for notational brevity.

A Lagrange function $\mathcal{L}_{\alpha_{k}}\left(\underline{s}_{\alpha_{k}}, \boldsymbol{u}_{\alpha_{k}}, \boldsymbol{\Lambda}_{\alpha_{k}}, \underline{\lambda}_{\alpha_{k}}\right) \equiv \mathcal{L}(\underline{s}, \boldsymbol{u}, \boldsymbol{\Lambda}, \underline{\lambda})$ related to problem P2(1) in (34) is formulated where the test function $\Lambda \in \mathscr{U}^{0}$ acts as a Lagrange multiplier for the equality constraint (34b), and the scalars $\underline{\lambda} \in \mathcal{S}^{2}$ act as Lagrange multipliers for the inequality constraints (34c)-(34d):

$$
\mathcal{L}(\underline{s}, \boldsymbol{u}, \boldsymbol{\Lambda}, \underline{\lambda}):=Q(\underline{s}, \boldsymbol{u})+G(\underline{s}, \boldsymbol{u} ; \boldsymbol{\Lambda})+\sum_{j=1}^{2 n_{s}} \lambda_{j} g_{j}(\underline{s}) \rightarrow \operatorname{stat}_{z \in \mathscr{Z}},
$$

where $(\underline{s}, \boldsymbol{u}, \boldsymbol{\Lambda}, \underline{\lambda})=: z \in \mathscr{Z}=: \mathcal{S} \times \mathscr{U} \times \mathscr{U}^{0} \times \mathcal{S}^{2}$. The quadruple $(\underline{s}, \boldsymbol{u}, \boldsymbol{\Lambda}, \underline{\lambda})$ is a local solution of the optimization minimum problem (34) provided the following 
Karush-Kuhn-Tucker conditions hold [Luenberger 1984]:

$$
\begin{array}{rlrl}
\mathcal{L}_{\underline{s}}^{\prime}(z ; \delta \underline{s}) & =Q_{\underline{s}}^{\prime}(\underline{s}, \boldsymbol{u} ; \delta \underline{s})+G_{\underline{s}}^{\prime}(\underline{s}, \boldsymbol{u} ; \boldsymbol{\Lambda}, \delta \underline{s})+\sum_{j=1}^{2 n_{s}} \lambda_{j} \nabla_{\underline{s}} g_{j}(\underline{s}) \cdot \delta \underline{s}=0 \\
& \text { for all } \delta \underline{s} \in \mathcal{S}, \\
\mathcal{L}_{\boldsymbol{u}}^{\prime}(z ; \delta \boldsymbol{u}) & =Q_{\underline{u}}^{\prime}(\underline{s}, \boldsymbol{u} ; \delta \boldsymbol{u})+G_{\boldsymbol{u}}^{\prime}(\underline{s}, \boldsymbol{u} ; \boldsymbol{\Lambda}, \delta \boldsymbol{u})=0 & \text { for all } \delta \boldsymbol{u} \in \mathscr{U}^{0}, \\
\mathcal{L}_{\Lambda}^{\prime}(z ; \delta \boldsymbol{\Lambda}) & =G_{\Lambda}^{\prime}(\underline{s}, \boldsymbol{u} ; \boldsymbol{\Lambda}, \delta \boldsymbol{\Lambda})=a(\underline{s}, \boldsymbol{u} ; \delta \boldsymbol{\Lambda})-\bar{l}(\delta \boldsymbol{\Lambda})=0 & \\
\frac{\partial \mathcal{L}(\boldsymbol{z})}{\partial \lambda}=g_{j} & \leq 0, \quad j=1, \ldots, 2 n_{s}, & \text { for all } \delta \boldsymbol{\Lambda} \in \mathscr{U}^{0}, \\
\lambda_{j} & \geq 0, & j=1, \ldots, 2 n_{s}, \\
\lambda_{j} g_{j} & =0, & j=1, \ldots, 2 n_{s}, &
\end{array}
$$

where $\delta \boldsymbol{z} \in \mathscr{Z}^{0}=: \mathcal{S} \times \mathscr{U}^{0} \times \mathscr{U}^{0} \times \mathcal{S}^{2}$. Equation (36a) is the gradient equation, (36b) is the costate (or adjoint) equation, (36c)-(36d) are state relations for primal admissibility, (36e) is a state relation for dual admissibility, and (36f) is a complementary optimality condition. Note that (36c) is nothing else but the state problem (6).

For a second-order sufficient optimality condition for a stationary point of the Lagrange function (35), we refer to [Luenberger 1984].

Alternatively to the direct differentiation method in Section 4.2 the first derivative of the quantity of interest $q^{\prime}(\underline{s} ; \delta \underline{s})$ is derived by exploiting the adjoint equation (36b). Setting $\delta \boldsymbol{u}=\bar{\delta} \boldsymbol{u}$ in (36b) and using the result (29a) with the choice $\delta \boldsymbol{u}=\boldsymbol{\Lambda}$ we obtain

$$
Q_{\boldsymbol{u}}^{\prime}(\underline{s}, \boldsymbol{u} ; \bar{\delta} \boldsymbol{u})=-G_{\boldsymbol{u}}^{\prime}(\underline{s}, \boldsymbol{u} ; \boldsymbol{\Lambda}, \bar{\delta} \boldsymbol{u})=G_{\underline{s}}^{\prime}(\underline{s}, \boldsymbol{u} ; \boldsymbol{\Lambda}, \delta \underline{s}) \quad \text { for all } \delta \underline{s} \in \mathcal{S},
$$

where it is noteworthy to recall the relation $\bar{\delta} \boldsymbol{u}=\boldsymbol{u}_{\underline{s}}^{\prime}(\underline{s} ; \delta \underline{s})$. Inserting the result (37) into (29b), the required result for the first derivative of the quantity of interest by the adjoint state method becomes

$$
q^{\prime}(\underline{s} ; \delta \underline{s})=Q_{\underline{s}}^{\prime}(\underline{s}, \boldsymbol{u}(\underline{s}) ; \delta \underline{s})+G_{\underline{s}}^{\prime}(\underline{s}, \boldsymbol{u}(\underline{s}) ; \boldsymbol{\Lambda}, \delta \underline{s}) \quad \text { for all } \delta \underline{s} \in \mathcal{S} .
$$

Furthermore, by comparing (38) to (36a) we observe

$$
\mathcal{L}_{\underline{s}}^{\prime}(z ; \delta \underline{s})=q^{\prime}(\underline{s} ; \delta \underline{s})+\sum_{j=1}^{2 n_{s}} \lambda_{j} \nabla_{\underline{s}} g_{j}(\underline{s}) \cdot \delta \underline{s}=\underline{0} \quad \text { for all } \delta \underline{s} \in \mathcal{S} .
$$


For the case of simple constraints (34c) and (34d), the relation (39) can be simplified. Upon taking the derivatives of the inequalities (34c) and (34d), we obtain

$$
\begin{aligned}
g_{j} & =s_{j, \alpha_{k}}^{L}-s_{j} \Longrightarrow \frac{d g_{j}}{d s_{j}}=-1 \\
& \Rightarrow \lambda_{j} \nabla_{\underline{s}} g_{j}=\left[0, \ldots,-\lambda_{j}, \ldots, 0\right]^{T} \in \mathbb{R}^{n_{s}}, \quad j=1, \ldots, n_{s}, \\
g_{n_{s}+j} & =s_{j}-s_{j, \alpha_{k}}^{R} \Longrightarrow \frac{d g_{n_{s}+j}}{d s_{j}}=1 \\
& \Longrightarrow \lambda_{n_{s}+j} \nabla_{\underline{s}} g_{n_{s}+j}=\left[0, \ldots, \lambda_{n_{s}+j}, \ldots, 0\right]^{T} \in \mathbb{R}^{n_{s}}, \quad j=1, \ldots, n_{s} .
\end{aligned}
$$

Consequently, exploiting the Karush-Kuhn-Tucker conditions (36e)-(36f), as well as the relations (30), the necessary optimality condition (39) decomposes into the relations

$$
\{\nabla q(\underline{s})\}_{i}=\frac{\partial q}{\partial s_{i}}=\left\{\begin{aligned}
& 0 \text { if } s_{i, \alpha_{k}}^{L}<s_{i}<s_{i, \alpha_{k}}^{R}, \\
&-\lambda_{i} \nabla_{\underline{s}} g_{i} \underline{e}_{i}=\lambda_{i} \geq 0 \quad \text { if } s_{i}=s_{i, \alpha_{k}}^{L}, \\
&-\lambda_{n_{s}+i} \nabla_{\underline{s}} g_{n_{s}+i} \underline{e}_{i}=-\lambda_{n_{s}+i} \leq 0 \quad \text { if } s_{i}=s_{i, \alpha_{k}}^{R},
\end{aligned}\right.
$$

which are identical to the optimality conditions (31).

To summarize, under the assumptions that $\boldsymbol{u}$ is a unique solution of the state problem (6) for given design variables $\underline{s}$ and $(\underline{s}, \boldsymbol{u}) \in \mathcal{S} \times \mathscr{U}$ is a local solution of the optimization problem (34), there exist Lagrange multipliers $\boldsymbol{\Lambda} \in \mathscr{U}^{0}$ and $\underline{\lambda} \in \mathcal{S}^{2}$, such that the quadruple $(\underline{s}, \boldsymbol{u}, \boldsymbol{\Lambda}, \underline{\lambda})$ satisfies the Karush-Kuhn-Tucker conditions (36) with Lagrange functional defined in (35).

A second-order sensitivity analysis by means of the adjoint state differentiation method is performed in Appendix A.2.

4.4. Relation of least-squares problems to $\alpha$-level optimization. As pointed out in Section 2.2, the least-squares functional in (17) is typically used for parameter identification, thus minimizing the model error in (16) in a least-squares sense. The corresponding optimization problem reads

$$
\text { Find } q_{\mathrm{LS}}^{*}=\min _{\underline{s} \in \mathcal{S}} q_{\mathrm{LS}}(\underline{s})=\min _{\underline{s} \in \mathcal{S}} Q_{\mathrm{LS}}(\underline{s}, \boldsymbol{u}(\underline{s}))
$$

with $q_{\mathrm{LS}}$ according to (17). The solution vector of the least-squares problem shall be denoted by $s^{*}=\left[s_{1}^{*}, \ldots, s_{i}^{*}, \ldots s_{n_{p}}^{*}\right]^{T}$. In the context of parameter identification the design space is a parameter space $\mathcal{S} \subset \mathbb{R}^{n_{p}}$ of $n_{p}$ physically admissible material parameters. For example, using Young's modulus and Poisson's ratio as independent material parameters in linear elasticity we have $\underline{s}=[E v], n_{p}=2, \mathcal{S}_{1}=\mathbb{R}_{+}$, $\mathcal{S}_{2}=[0,0.5]$, and $\mathcal{S}=\mathcal{S}_{1} \times \mathcal{S}_{2} \subset \mathbb{R}^{2}$.

For the fuzzy analysis of the goal function $q_{\mathrm{LS}}$ we construct membership functions as triangular fuzzy numbers according to (21) by means of the least-squares 

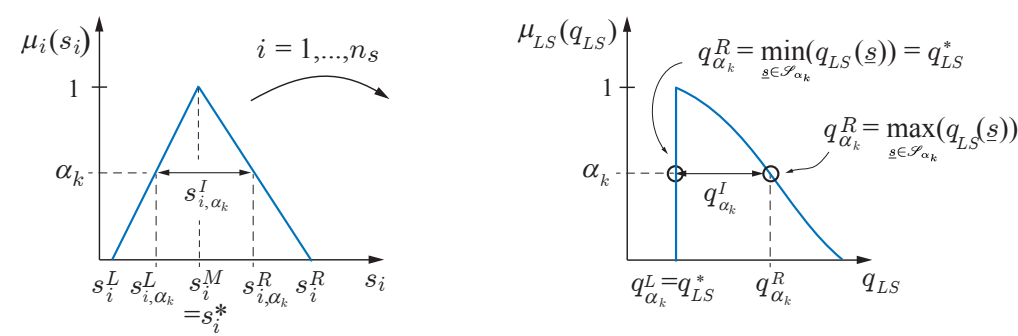

Figure 3. Schematic graph of $\alpha$-level optimization for leastsquares problems. Left: input membership function. Right: output membership function.

solutions $s_{i}^{*}$ as

$$
\begin{aligned}
\hat{A}_{i}=\left\langle s_{i}^{L}, s_{i}^{M}, s_{i}^{R}\right\rangle=\left\langle f^{L} s_{i}^{*}, s_{i}^{*}, f^{R} s_{i}^{*}\right\rangle, \\
\quad \text { where } f^{L}<1, f^{R}>1 \text {, and } i=1, \ldots, n_{s} .
\end{aligned}
$$

This is illustrated in Figure 3, left. For the corresponding $\alpha$-discretization we obtain lower and upper bounds of the crisp intervals $\mathcal{S}_{i, \alpha_{k}}=\left[s_{i, \alpha_{k}}^{L}, s_{i, \alpha_{k}}^{R}\right]$ at level $\alpha_{k}$ according to (23). With the corresponding input membership functions $\mu_{i}\left(s_{i}\right)$ it is the goal to determine the output membership function $\mu_{\mathrm{LS}}\left(q_{\mathrm{LS}}\right)$ of the quantity of interest $q_{\mathrm{LS}}$.

Since $s^{*}$ minimizes the least-squares problem (42), and since by construction of (43) we have $s_{i}^{*} \in \mathcal{S}_{i, \alpha_{k}}$, the minimum problems in (25) render the same solution $s^{*}$ to

$$
q_{\alpha_{k}}^{L}=\min _{\underline{s} \in \mathcal{S}_{\alpha_{k}}} q(\underline{s})=q_{\mathrm{LS}}^{*}=\min _{\underline{s} \in \mathcal{S}} q_{\mathrm{LS}}(\underline{s}) \quad \text { for all } 0 \leq \alpha_{k} \leq 1
$$

that is independent of the $\alpha$-level. Please note also that none of the constraints (23) in the minimization problems (44) are activated, that is, they are unconstrained. Consequently, the result (44) renders a vertical line for the left-hand part of the membership function in Figure 3, right. On the other hand, the maximization values

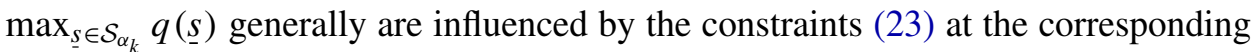
$\alpha$-levels, thus resulting in a decreasing part in the right-hand part of the membership function, which is schematically illustrated in Figure 3, right.

\section{Finite element discretization}

We introduce a finite element (FE) discretization as $\underline{s}_{h} \in \mathcal{S}_{h, \alpha_{k}} \subset \mathcal{S}_{\alpha_{k}}, \boldsymbol{u}_{h} \in \mathscr{U}_{h} \subset \mathscr{U}$, where $\mathcal{S}_{h, \alpha_{k}}$ and $\mathscr{U}_{h}$ are appropriate finite element spaces. In most cases, due to the finite dimension of $\mathcal{S}_{\alpha_{k}}$, the design variables $\underline{s}_{h}$ are in the same space as $\underline{s}$. An exception is the concept of hierarchical modeling; see, e.g., [Johansson et al. 2008]. 
The FE-discretized version of problem (25) is: find $\underline{s}_{h} \in \mathcal{S}_{\alpha_{k}}$ such that

$$
q\left(\underline{s}_{h}\right)=Q\left(\underline{s}_{h}, \boldsymbol{u}_{h}\left(\underline{s}_{h}\right)\right) \rightarrow \min _{\underline{s}_{h} \in \mathcal{S}_{h, \alpha_{k}}} \text { and } \quad G\left(\underline{s}_{h}, \boldsymbol{u}_{h}\left(\underline{s}_{h}\right)\right)=0 .
$$

In addition, with the discretization $\boldsymbol{\Lambda}_{h} \in \mathscr{U}_{h}^{0} \subset \mathscr{U}^{0}$ the FE-discretized version of the Lagrange function (35) is

$$
\mathcal{L}\left(\underline{s}_{h}, \boldsymbol{u}_{h}, \boldsymbol{\Lambda}_{h}, \underline{\lambda}_{h}\right):=Q\left(\underline{s}_{h}, \boldsymbol{u}_{h}\right)+G\left(\underline{s}_{h}, \boldsymbol{u}_{h} ; \boldsymbol{\Lambda}_{h}\right)+\sum_{i=1}^{2 n_{s}} \lambda_{h, j} g_{j}\left(\underline{s}_{h}\right) \underset{ }{\rightarrow} \operatorname{stat}_{z_{h} \in \mathscr{Z}_{h}},
$$

where $\left(\underline{s}_{h}, \boldsymbol{u}_{h}, \boldsymbol{\Lambda}_{h}, \underline{\lambda}_{h}\right)=: z_{h} \in \mathscr{Z}_{h}=: \mathcal{S}_{h} \times \mathscr{U}_{h} \times \mathscr{U}_{h}^{0} \times \mathcal{S}_{h}^{2}$ has been introduced. The quadruple $\left(\underline{s}_{h}, \boldsymbol{u}_{h}, \boldsymbol{\Lambda}_{h}, \underline{\lambda}_{h}\right)=: \boldsymbol{z}_{h}$ solves the discretized form of the optimization problem P2 in (34) provided a discretized form of the Karush-Kuhn-Tucker relations (36) is satisfied.

\section{Optimization methods}

Algorithms for solution of the optimization problems (25a) and (45) may be grouped into methods where only function evaluations are needed (zero-order methods) and where in addition its gradients are required (first-order methods), respectively. Another classification into deterministic and stochastic methods is possible. Examples of gradient-based deterministic methods are the Gauss-Newton method, the Levenberg-Marquard method, or the BFGS method; see, e.g., [Dennis and Schnabel 1983; Luenberger 1984]. An example of a gradient-free deterministic method is the simplex method; see, e.g., [Press et al. 1992]. Examples for stochastic methods are the Monte Carlo method, the evolution strategy, the method of Price and the method of Müller and Nollau; see, e.g., [Schwefel 1977], and [Möller et al. 2000] in the context of $\alpha$-level optimization.

Starting with an initialization $\underline{s}_{h}^{(0)}$, solution of the minimization problem (45) is achieved iteratively with a deterministic gradient-based strategy:

$$
\begin{aligned}
& \text { Projection algorithm [Bertsekas 1982]. } \\
& \underline{s}^{(j+1)}=\mathcal{P}\left\{\underline{s}^{(j)}-\alpha^{(j)} \underline{\bar{H}}^{(j)} \nabla q\left(\underline{s}^{(j)}\right)\right\} \\
& \text { where }(\mathcal{P}\{\underline{s}\})_{i}:=\min \left(s_{i, \alpha_{k}}^{L}, \max \left(s_{i}, s_{i, \alpha_{k}}^{R}\right)\right), i=1, \ldots, n_{s} .
\end{aligned}
$$

According to (23), $s_{i, \alpha_{k}}^{L}, s_{i, \alpha_{k}}^{R}$ are lower and upper bounds for the design variables. The iteration procedure (47) ensures a descent of the function value and feasibility of the parameters at each iteration step $j$. With the Jacobian $\underline{J}$ defined in (32b), 
three examples of iteration matrices at the $j$-th iteration step are

$$
\begin{aligned}
\text { Newton } & \underline{H}_{\mathrm{N}} & =\left[\nabla^{2} q(\underline{s})\right]^{-1}, \\
\text { Gauss-Newton } & \underline{H}_{\mathrm{GN}} & =\left[\underline{J}^{T} \underline{W}^{T} \underline{W} \underline{J}\right]^{-1}, \\
\text { Levenberg-Marquardt } & \underline{H}_{\mathrm{LM}} & =\left[\underline{J}^{T} \underline{W}^{T} \underline{W} \underline{J}+\gamma \boldsymbol{I}\right]^{-1}, \\
\text { BFGS } & \underline{H}_{\mathrm{BFGS}} & =\underline{H}\left(\underline{H}^{(j-1)}, \nabla q, \underline{s}\right) .
\end{aligned}
$$

The Newton method uses the full Hessian in (33), whereas the Gauss-Newton method neglects the matrix $\underline{M}$ in (33). It is not suitable if $\underline{J}$ does not have full rank. Then a Levenberg-Marquardt matrix becomes preferable, where various versions can be distinguished applying different strategies to choose the parameter $\gamma$; see, e.g., [Dennis and Schnabel 1983; Luenberger 1984]. Concerning the update formula for the BFGS algorithm we refer to [Luenberger 1984]. In order to preserve the descent property, a so-called diagonalization $\underline{H} \rightarrow \underline{\bar{H}}$ according to [Bertsekas 1982; Mahnken 1993] is employed. The line-search parameter $\alpha^{(j)}$ in the scheme (47) enlarges the area of convergence. An alternative globalization technique is trust-region; see, e.g., [Nocedal and Wright 1999].

Note that the above scheme (47) requires solution of the discrete version of the state problem (6). For nonlinear problems this is achieved within inner (equilibrium) iteration loops by means of the discrete counterpart of the Newton method in the equations (8). In addition, $n_{s}$ discrete versions of the tangent problem in (29a) have to be solved for calculation of the gradient $\nabla q$ in (32). To this end, the same (generally unsymmetric) linear tangent $G_{\boldsymbol{u}}^{\prime}(\cdot, \cdot ; \cdot, \cdot)$ as in (8), but with different right-hand sides, is used.

Gradient-based methods are able to determine only local minima, however are unable to overcome these. Therefore, different starting values should be applied for these types of methods. Stochastic methods can overcome local minima, however very often are not effective with respect to convergence. The advantages of both methods can be exploited in a hybrid strategy; see, e.g., [Quagliarella and Vicini 1998].

Similarly, solution of the discrete form of the Karush-Kuhn-Tucker equations (36) is obtained with the assembled vectors

$$
\tilde{\tilde{s}}=\left[\begin{array}{c}
\underline{s}_{h} \\
\boldsymbol{u}_{h} \\
\boldsymbol{\Lambda}_{h}
\end{array}\right], \quad \underline{r}=\left[\begin{array}{c}
\nabla_{s} \mathcal{L} \\
\nabla_{\boldsymbol{u}} \mathcal{L} \\
\nabla_{\boldsymbol{\Lambda}} \mathcal{L}
\end{array}\right] .
$$

Starting with an initialization $\underline{\tilde{s}}^{(0)}$, similarly to (50), the iteration scheme is now $\left.\underline{\tilde{s}}^{(j+1)}=\mathcal{P}\left\{\underline{\tilde{s}}^{(j)}-\alpha^{(j)} \underline{\bar{H}}^{(j)} \underline{r}^{(j)}\right)\right\}$,

$$
\text { where }(\mathcal{P}\{\underline{\tilde{s}}\})_{i}:= \begin{cases}\min \left(s_{i, \alpha_{k}}^{L}, \max \left(s_{i}, s_{i, \alpha_{k}}^{R}\right)\right) & \text { if } i=1, \ldots, n_{s}, \\ (\mathcal{P}\{\underline{\tilde{s}}\})_{i}:=\tilde{s}_{i} & \text { if } i=n_{s}+1, \ldots, \operatorname{dim}(\underline{\tilde{s}}),\end{cases}
$$



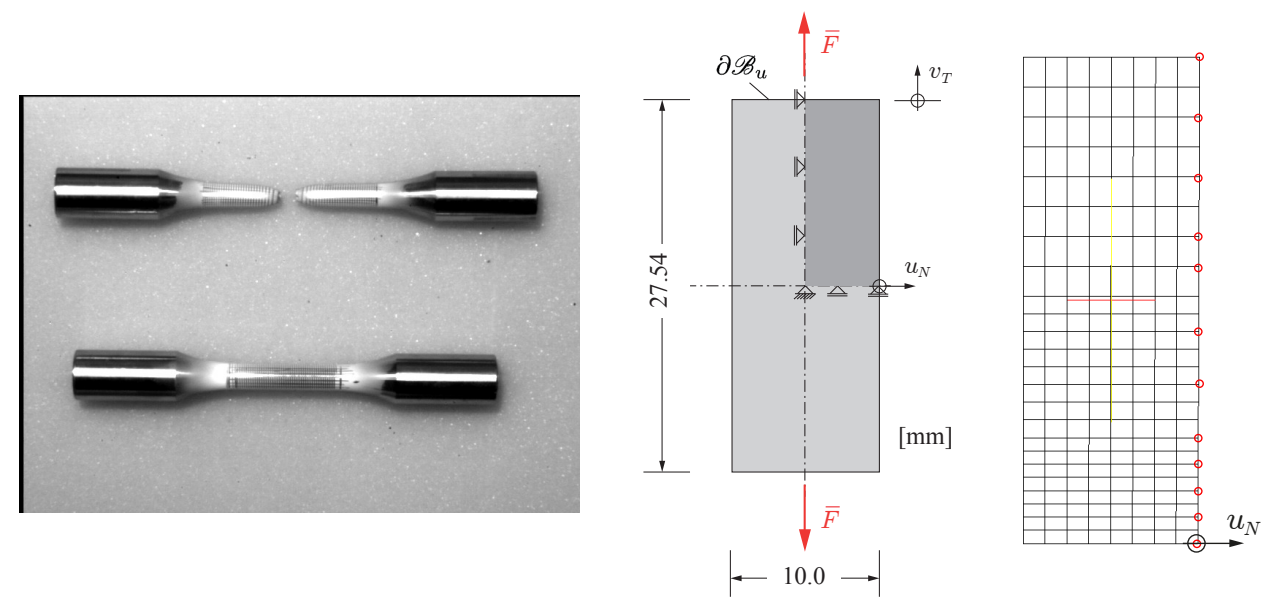

Figure 4. Axisymmetric necking. Left: photograph of failed and unused samples. Center: geometry. Right: discretization of a quarter with observation points.

until some convergence criterion is achieved. Contrary to the more conventional scheme (47), the iteration scheme (50) iterates simultaneously on all unknowns of the triple $\left(\underline{s}_{h}, \boldsymbol{u}_{h}, \boldsymbol{\Lambda}_{h}\right)$, thus avoiding inner (equilibrium) iteration loops. Note that the scheme (50) is an extension of the iterative scheme in [Johansson et al. 2007] for problems without simple constraints.

\section{Representative examples}

7.1. Axisymmetric necking at large strain elastoplasticity. In this example we consider the necking of a circular bar. The material of the specimen is a structural steel S355J2+N, according to EN 10025-2:200410 (formerly Baustahl St52-3 N, according to the German industrial code DIN 17100). In the photography in Figure 4, left, the sample after the experiment and a comparative sample are shown.

For experimental determination of the displacements, gratings are positioned on the surface. These are photographed with digital cameras at consecutive observation states NLST as listed in Table 1 in the displacement controlled experiment [Andresen et al. 1996]. The geometry under investigation is depicted in Figure 4, center. Here $v_{T}$ represents the total elongation at the different observation states with values according to Table 1 , and $\bar{F}$ is the resulting reaction force. Figure 4, right, shows a finite element discretization of a quarter model with selected observation points for parameter identification and the subsequent fuzzy analysis. Figure 5, top, depicts the grating at four observation states NLST $=5,7,10,13$ as introduced in Table 1. 


\begin{tabular}{|l|ccccc|}
\hline NLST & 1 & 3 & 4 & 5 & 6 \\
$v_{T}[\mathrm{~mm}]$ & $1.51 \cdot 10^{-3}$ & $1.2 \cdot 10^{-2}$ & $4.3 \cdot 10^{-2}$ & $1.1 \cdot 10^{-1}$ & $3.39 \cdot 10^{-1}$ \\
NLST & 7 & 8 & 10 & 13 & \\
$v_{T}[\mathrm{~mm}]$ & 3.24 & 3.83 & 4.61 & 5.47 & \\
\hline
\end{tabular}

Table 1. Axisymmetric necking: total elongation $v_{T}$ at 9 observation states.
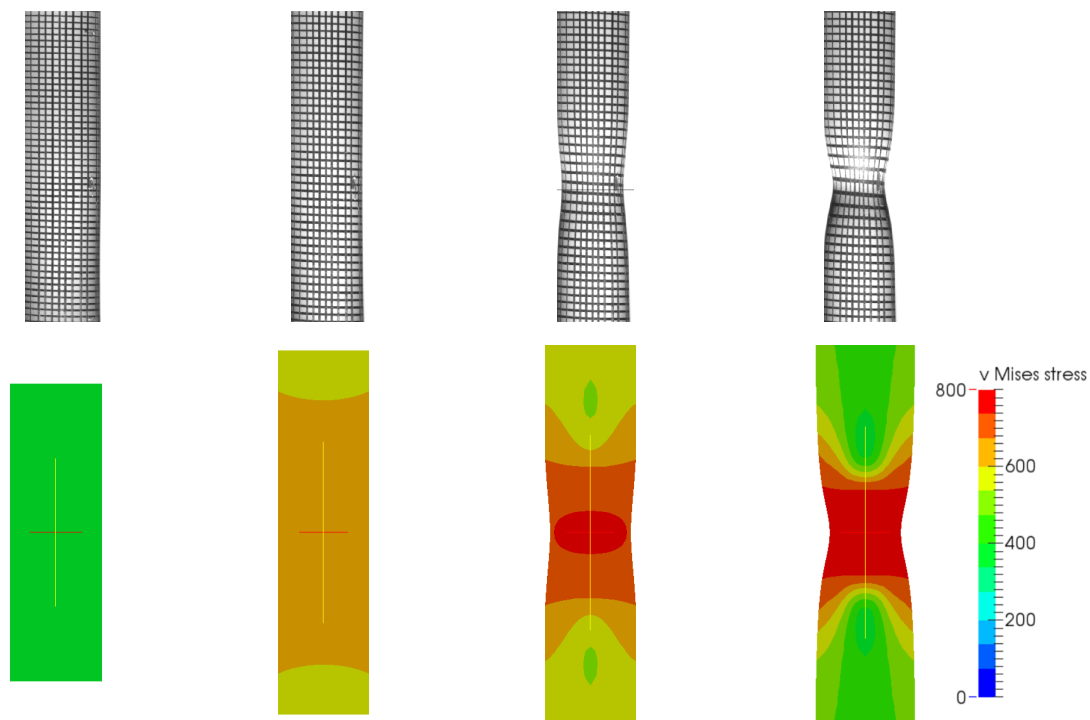

Figure 5. Axisymmetric necking. Top: photographs with a CCD camera of the sample and the grating at four different observation states NLST $=5,7,10,13$ according to Table 1 . Bottom: contours of von Mises stresses over the deformed configurations obtained from FE simulation.

The material is modeled by large strain multiplicative von Mises elastoplasticity according to

\begin{tabular}{|rl|}
\hline Kirchhoff stress & $\boldsymbol{\tau}=\mu \operatorname{dev}\left(\ln \boldsymbol{b}_{\mathrm{el}}\right)+K \ln J \boldsymbol{g}^{\sharp}$, \\
Yield function & $\Phi(\boldsymbol{\tau}, e ; \underline{\kappa})=\|\operatorname{dev}(\boldsymbol{\tau})\|-\sqrt{\frac{2}{3}} Y(e ; \underline{\kappa})$, \\
Flow stress & $Y(e ; \underline{\kappa})=Y_{0}+q(1-\exp (-b e))$, \\
Flow rule & $-\frac{1}{2} \mathcal{L}_{t}\left(\boldsymbol{b}_{\mathrm{el}}\right) \boldsymbol{b}_{\mathrm{el}}^{-1}=\gamma \frac{\operatorname{dev}(\boldsymbol{\tau})}{\|\operatorname{dev}(\boldsymbol{\tau})\|}$, \\
Variable evolution & $\dot{e}=\gamma \sqrt{\frac{2}{3}}$, \\
Loading and unloading conditions & $\gamma \geq 0, \Phi \leq 0, \gamma \Phi=0$. \\
\hline
\end{tabular}


Here, in addition to the notation in Section 2, we use $\tau=J \sigma$ as the Kirchhoff stress tensor with determinant $J>0$ of (4b), $\boldsymbol{b}_{\mathrm{el}}=\boldsymbol{F}_{\mathrm{el}} \boldsymbol{F}_{\mathrm{el}}^{T}$ as the elastic left Cauchy-Green tensor based on the multiplicative decomposition $\boldsymbol{F}=\boldsymbol{F}_{\mathrm{el}} \boldsymbol{F}_{\mathrm{pl}}$ for the deformation gradient in (4a), and $\boldsymbol{g}^{\sharp}$ as the contravariant metric tensor, and $\mathcal{L}_{t}(\cdot)={ }_{*} \Phi_{e}^{\sharp}\left[\partial_{t}^{*} \Phi_{e}^{\sharp}(\cdot)\right]$ denotes the Lie (time) derivative of a spatial argument with elastic pull-back and push-forward operators ${ }^{*} \Phi_{e}^{\sharp},{ }_{*} \Phi_{e}^{\sharp}$ according to (B-4) with $\boldsymbol{F}_{\mathrm{el}}$ replaced by $\boldsymbol{F}$; see, e.g., [Marsden and Hughes 1994; Simo and Miehe 1992; Mahnken 2005]. The vector of material parameters characterizing the inelastic material behavior is defined as

$$
\underline{s}=\left[Y_{0} b q\right]^{T} .
$$

Due to obvious symmetry conditions only a quarter of the specimen in Figure 4, right, is considered in the finite element simulation. An axisymmetric strain and stress state is assumed in the calculations. The mixed finite element formulation is based on a four-noded geometrically nonlinear enhanced strain method according to [Simo and Armero 1992]. A detailed analysis for the related sensitivity terms of the direct and the inverse analysis is provided in [Mahnken and Stein 1997].

The elastic constants are predetermined as $E=20600 \mathrm{kN} / \mathrm{cm}^{2}$ for Young's modulus and $v=0.3$ for Poisson's ratio. The additional 3 material parameters in (52) characterizing the inelastic material behavior are obtained by minimizing the least-squares functional

$$
q_{\mathrm{LS}}(\underline{s})=Q_{\mathrm{LS}}(\boldsymbol{u}(\underline{s}))=\sum_{i=1}^{n_{\mathrm{tdat}}} \sum_{j=1}^{n_{\mathrm{mp}}}\left\|\underline{\boldsymbol{u}}_{i j}(\underline{s})-\underline{\bar{u}}_{i j}\right\|+\sum_{i=1}^{n_{\mathrm{tdat}}}\left(w\left(F_{i}(\underline{s})-\bar{F}_{i}\right)\right) .
$$

Here $\underline{\boldsymbol{u}}_{i j}=\left[\bar{u}_{x}, \bar{u}_{y}\right]_{i j}$ and $\bar{F}_{i}$ assemble experimental data for displacements at $n_{\mathrm{mp}}$ observation points and reaction forces, respectively, for $n_{\text {tdat }}$ observation states. Analogously, $\underline{\boldsymbol{u}}_{i j}=\left[u_{x}, u_{y}\right]_{i j}$ assembles simulated data for displacements and $F_{i}(\underline{s})$ denotes reaction forces, analogously to the quantity of interest in (10), i.e.,

$$
F_{i}(\boldsymbol{u})=\int_{\partial \mathscr{B}_{u}}\left\|\boldsymbol{P}\left(\boldsymbol{u}_{i}\right)^{T} \cdot \boldsymbol{N}\right\|_{2} d A, \quad i=1, \ldots, n_{\text {tdat }}
$$

To be more specific, the weighting factor in (53) is chosen as $w=\sqrt{10^{-3}}$, the number of load steps is $N=40$, the number of observation states is $n_{\text {tdat }}=9$ according to Table 1 , and there are $n_{\mathrm{mp}}=12$ observation points. In this way, the functional (53) represents a special case of (17) with the quantity of interest for the model error by taking into account both displacements and reaction forces with adequate weighting. 

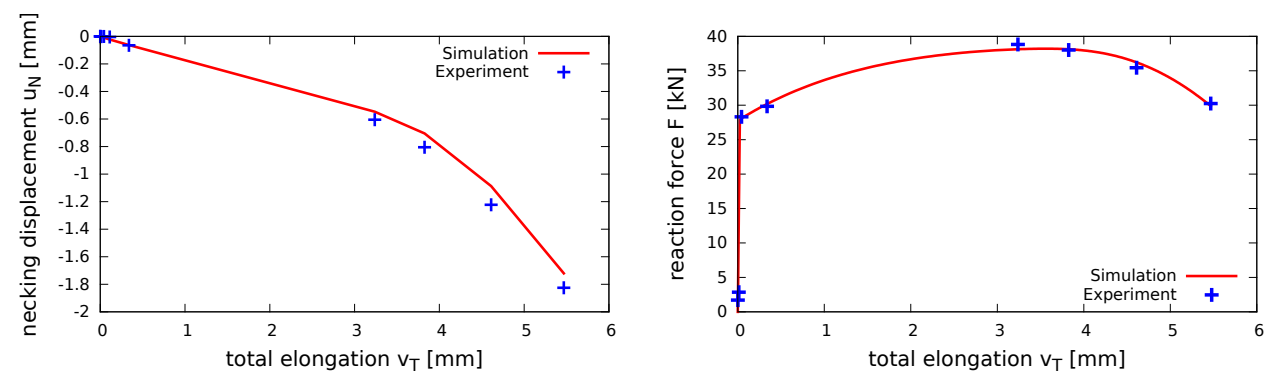

Figure 6. Axisymmetric necking: comparison of simulation and experiment. Left: necking displacement versus loading. Right: reaction force versus loading.
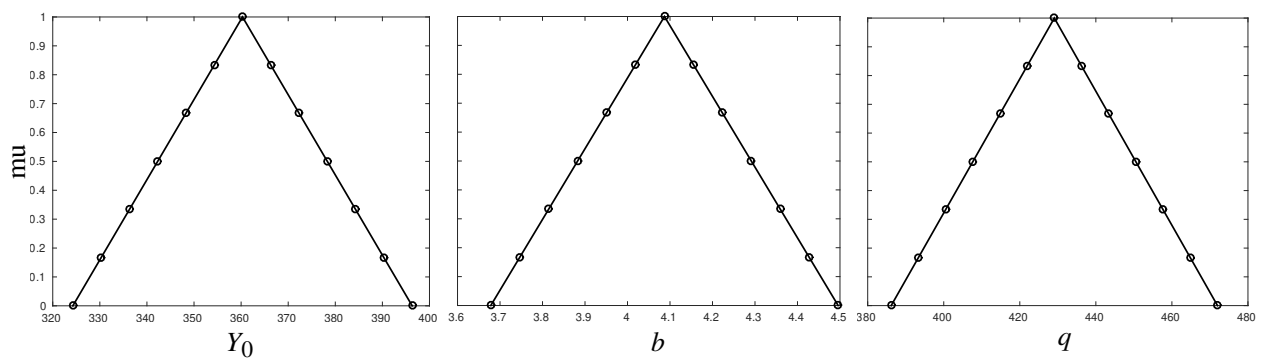

Figure 7. Axisymmetric necking: input membership functions for the material parameters $Y_{0}, b, q$.

The resulting vector for the material parameters after optimization is

$$
\underline{s}^{*}=\left[\begin{array}{c}
Y_{0} \\
b \\
q
\end{array}\right]=\left[\begin{array}{c}
360.30 \mathrm{~N} / \mathrm{mm}^{2} \\
4.0869 \\
429.08 \mathrm{~N} / \mathrm{mm}^{2}
\end{array}\right] .
$$

Figure 5, bottom, depicts the von Mises stress over the deformed configurations. The nonuniformness with increasing necking behavior becomes striking. In Figure 6, left and right, the maximal necking displacement $u_{N}$, introduced in Figure 4, center, and the total load $F$, defined in (54), respectively, versus the total elongation $v_{T}$ are compared for FE simulation and experimentation, thus illustrating the excellent agreement for both quantities.

Based on the above results of parameter identification, the following considerations are directed to the fuzzy analysis of the material parameters obtained in (55). To this end, firstly membership functions are generated according to Figure 7 as input quantities for the fuzzy analysis. The parameters $Y_{0}, b, q$ in (52) are chosen as mean values to obtain triangular fuzzy numbers according to (44) as

$$
\hat{A}_{i}=\left\langle s_{i}^{L}, s_{i}^{M}, s_{i}^{R}\right\rangle=\left\langle 0.9 s_{i}^{*}, s_{i}^{*}, 1.1 s_{i}^{*}\right\rangle,
$$



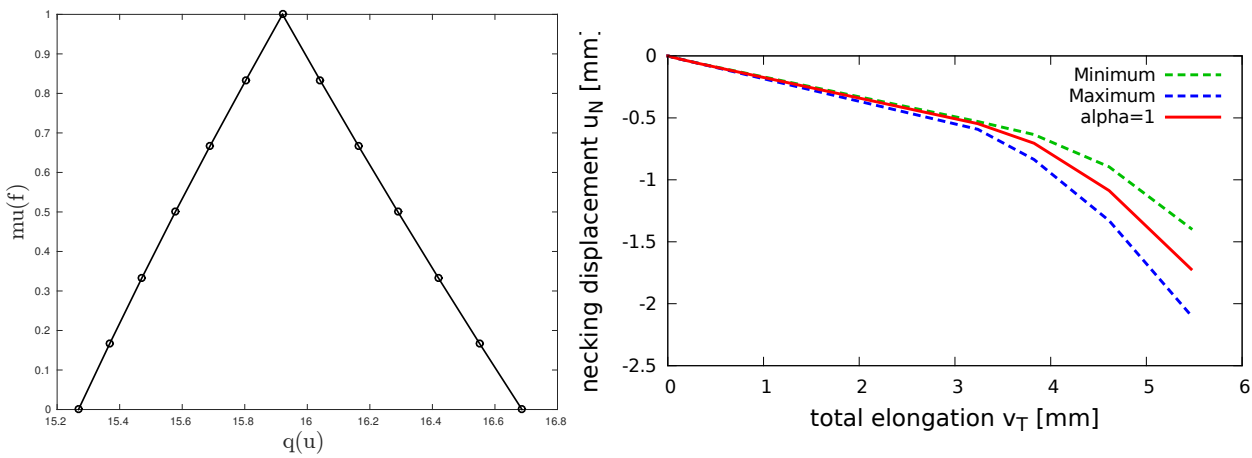

Figure 8. Axisymmetric necking: fuzzy results for quantity of interest with displacements. Left: output membership function. Right: uncertainty regime.
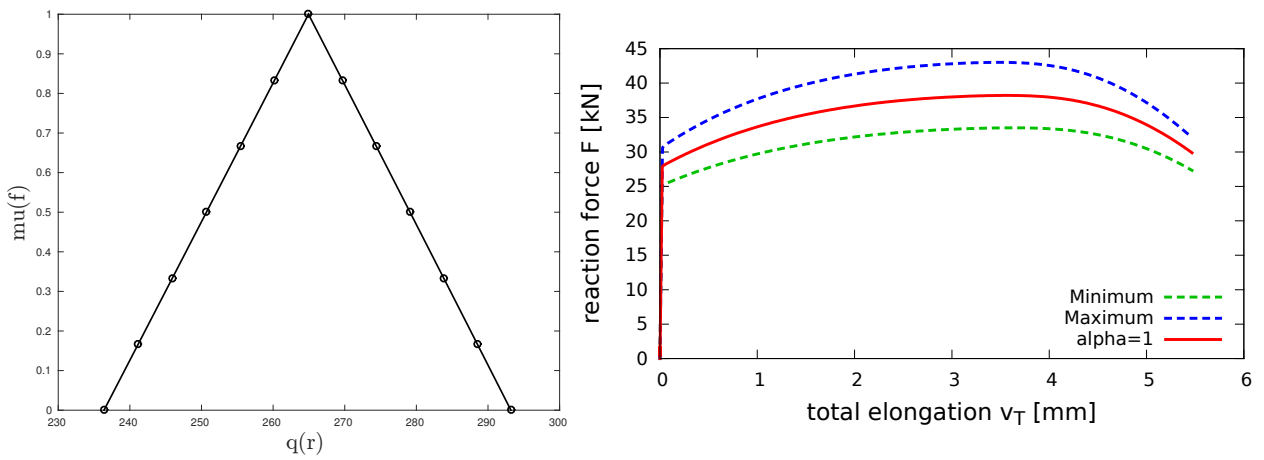

Figure 9. Axisymmetric necking: fuzzy results for quantity of interest with reaction forces. Left: output membership function. Right: uncertainty regime.

that is, for each material parameter a maximum deviation of $10 \%$ for the uncertainty is assumed.

The $\alpha$-discretization is performed with 7 equally spaced intervals. In the fuzzy analysis we are interested in the uncertainties of the quantities of interest

$$
\begin{aligned}
& q_{u}(\underline{s}):=Q(\boldsymbol{u}(\underline{s})):=\sum_{i=1}^{n_{\text {tdat }}} \sum_{j=1}^{n_{\mathrm{mp}}}\left\|\underline{\boldsymbol{u}}_{i j}(\underline{s})\right\|, \\
& q_{F}(\underline{s}):=Q\left(F_{i}(\underline{s})\right):=\sum_{i=1}^{n_{\text {tdat }}} F_{i}(\underline{s})
\end{aligned}
$$

with displacements $\underline{\boldsymbol{u}}_{i j}(\underline{s})$ according to (53) and reaction forces $F_{i}(\underline{s})$ according to (54). As a third quantity we choose the model error functional $q_{\mathrm{LS}}(\underline{s})$ in (53). 

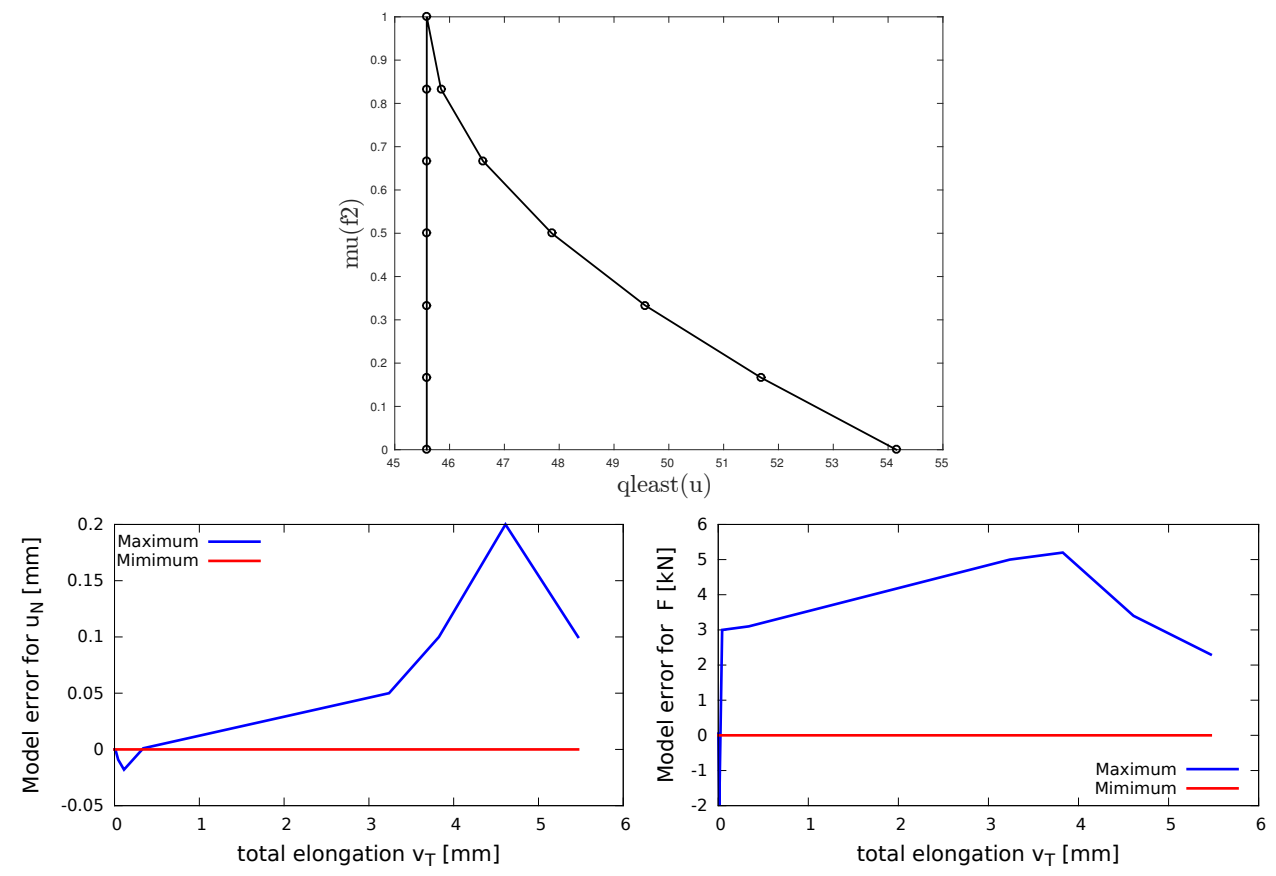

Figure 10. Axisymmetric necking: fuzzy results for quantity of interest with model error. Top: output membership function. Bottom left: uncertainty regime for model of necking displacement. Bottom right: uncertainty regime for model of reaction force.

For all three quantities of interest the minimization and maximization problems P1a and P1b, respectively, according to (25) are solved at each $\alpha$-level.

Figures 8 and 9 , left, show the output membership functions $\mu\left(q_{u}\right)$ and $\mu\left(q_{F}\right)$, respectively, for the quantity of interest with displacements in (57a) and the quantity of interest with reaction forces in (57b). Remarkably, both output membership functions exhibit an almost linear behavior, although the underlying geometrical and physical behavior is highly nonlinear. The corresponding Figures 8 and 9, right, display the uncertainty regimes for the necking displacement $u_{N}$ and the reaction force $\bar{F}$.

Figure 10 summarizes the fuzzy results for the quantity of interest formulated as a model error based on the least-squares functional in (53). Figure 10, top, shows the output membership functions $\mu\left(q_{\mathrm{LS}}\right)$. Note that the minimum problems $\min _{s \in \mathcal{S}_{\alpha_{k}}} q(s)$ in (25) of all $\alpha$-level optimization coincide with the minimization problems for the least-squares functional in (53). As explained in Section 4.4, the constraints (23) are not activated at the $\alpha$-levels, thus resulting in a vertical line in the left-hand side of the membership function in Figure 10, top. On the other 
hand, the maximization values $\max _{s \in \mathcal{S}_{\alpha_{k}}} q(s)$ exhibit a strong nonlinear behavior in Figure 10, top, influenced by the constraints (23) at the corresponding $\alpha$-levels. Figure 10, bottom left and right, display the related uncertainty regimes for the model errors of necking displacement and reaction force, respectively. Note that in both diagrams the curves obtained from the minimum problems coincide with those of $\alpha=1$.

The maximum deviations from the quantities at $\alpha=1$ for all three quantities are

$$
\begin{aligned}
& \Delta_{u}^{L}=\frac{q_{u \alpha=0}^{L}-q_{u \alpha=1}^{L}}{q_{u \alpha=1}^{L}} 100=4.10 \%, \quad \Delta_{u}^{R}=\frac{q_{u \alpha=0}^{R}-q_{u \alpha=1}^{L}}{q_{u \alpha=1}^{L}} 100=4.81 \%, \\
& \Delta_{F}^{L}=\frac{q_{F \alpha=0}^{L}-q_{F \alpha=1}^{L}}{q_{F \alpha=1}^{L}} 100=10.77 \%, \Delta_{F}^{R}=\frac{q_{F \alpha=0}^{R}-q_{F \alpha=1}^{L}}{q_{F \alpha=1}^{L}} 100=10.68 \% \text {, } \\
& \Delta_{\mathrm{LS}}^{L}=\frac{q_{\mathrm{LS} \alpha=0}^{L}-q_{\mathrm{LS} \alpha=1}^{L}}{q_{\mathrm{LS} \alpha=1}^{L}} 100=0 \%, \quad \Delta_{\mathrm{LS}}^{R}=\frac{q_{\mathrm{LS} \alpha=0}^{R}-q_{\mathrm{LS} \alpha=1}^{L}}{q_{\mathrm{LS} \alpha=1}^{L}} 100=18.79 \%,
\end{aligned}
$$

thus revealing a larger uncertainty for the reaction forces as for the displacements.

7.2. Perforated strip at large strain elasticity. In the second representative example measurements are obtained from an experiment for a polyurethane sample. The experimental setting is visualized in Figure 11, top left. The geometry and dimensions of the specimen are shown in Figure 11, top center, where $t=2 \mathrm{~mm}$ is its thickness. Measurements for the parameter identification are taken at prescribed displacements $v_{T}$ according to Table 2 . The values for $\bar{F}$ in Table 2 represent the measured total loads at the different observation states. Displacement fields are obtained by noncontacting optical measurements, which require a speckle pattern on the surface of the specimen as shown in Figure 11, top right. The observation points, where measurements are available, are highlighted in Figure 11, bottom.

The material is modeled in large strain elasticity with an the Ogden model according to [Ogden 1997]. This hyperelastic material model is based on principal stretches with a strain-energy function for the isochoric part given by

$$
\hat{\psi}(\boldsymbol{u}, \underline{s})=\sum_{A=1}^{3} \sum_{\alpha=1}^{n} \frac{c_{\alpha}}{m_{\alpha}}\left(\hat{\lambda}_{A}^{m_{\alpha}}-1\right) .
$$

The deviatoric principal stretches are given by $\hat{\lambda}_{A}=J^{-1 / 3} \lambda_{A}$ with principal stretches $\lambda_{A}$, obtained from a spectral decomposition of the right Cauchy-Green tensor $C=\sum_{A=1}^{3} \lambda_{A}^{2} N_{A} \otimes N_{A}$. The vector of material parameters is defined as

$$
\underline{s}=\left[c_{1}, m_{1}, \ldots, c_{n}, m_{n}\right]^{T} .
$$



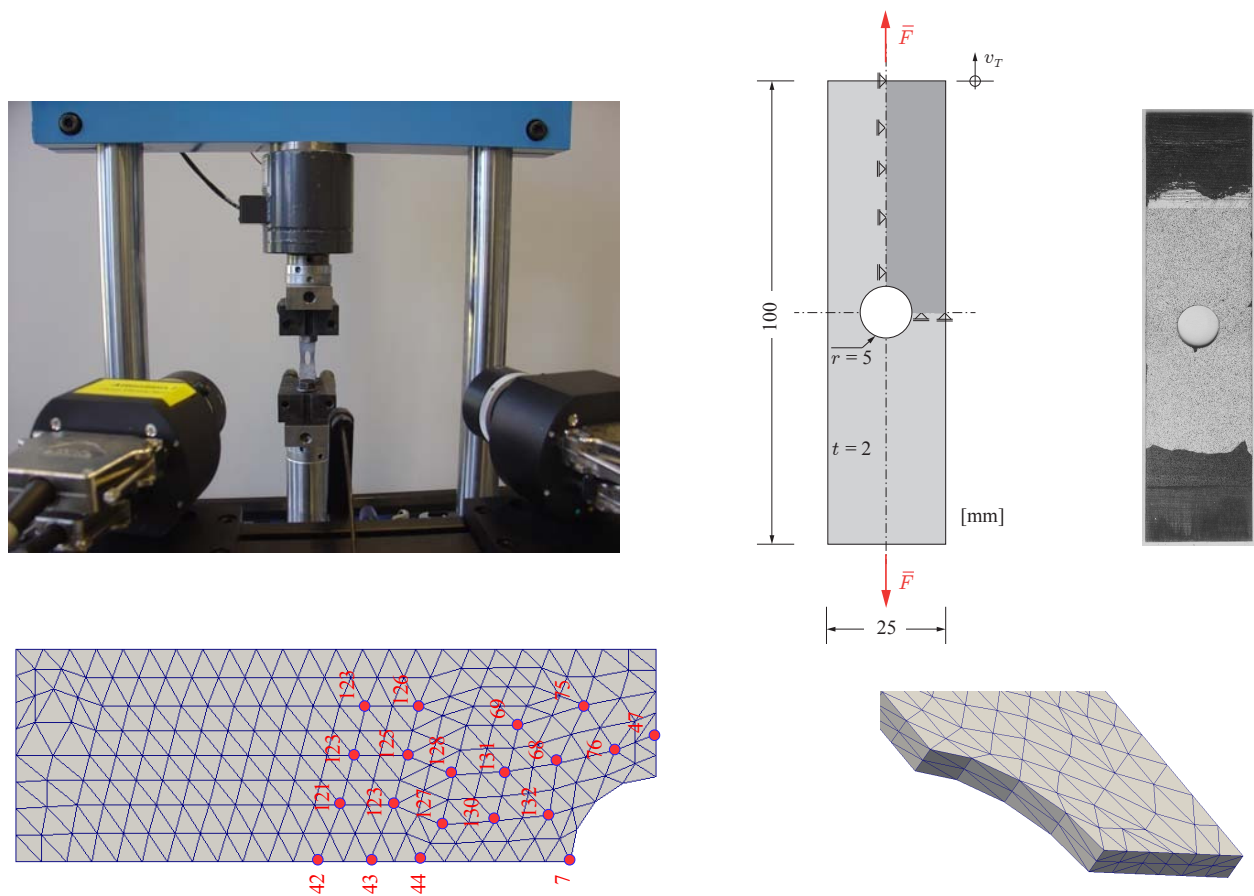

Figure 11. Perforated strip. Top left: experimental setup. Top center: geometry. Top right: speckle pattern. Bottom: threedimensional discretization of a quarter with observation points.

\begin{tabular}{|l|lllll|}
\hline NLST & 6 & 12 & 18 & 24 & 30 \\
$v_{T}[\mathrm{~mm}]$ & 6 & 12 & 18 & 24 & 30 \\
$\bar{F}[\mathrm{~N}]$ & 10.88 & 17.23 & 21.33 & 24.31 & 26.53 \\
\hline
\end{tabular}

Table 2. Perforated strip: total load $\bar{F}$ at 5 observation states.

Closed-form expressions for stresses and tangent moduli in terms of the reference configuration as well as the current configuration have been derived in [Simo and Taylor 1991].

Due to obvious symmetry conditions only a quarter of the specimen in Figure 11, bottom, is considered in the finite element simulation. The mixed finite element formulation is based on a four-noded geometrically nonlinear enhanced strain method for tetrahedra according to [Caylak and Mahnken 2012]. A detailed analysis for the related sensitivity terms of the direct and inverse analyses is provided in [Mahnken and Stein 1997].

Hereafter, we set $n=1$ for the Ogden model in (60); that is, $c_{1}$ and $m_{1}$ are the required material parameters. The finite element simulation is force-driven 


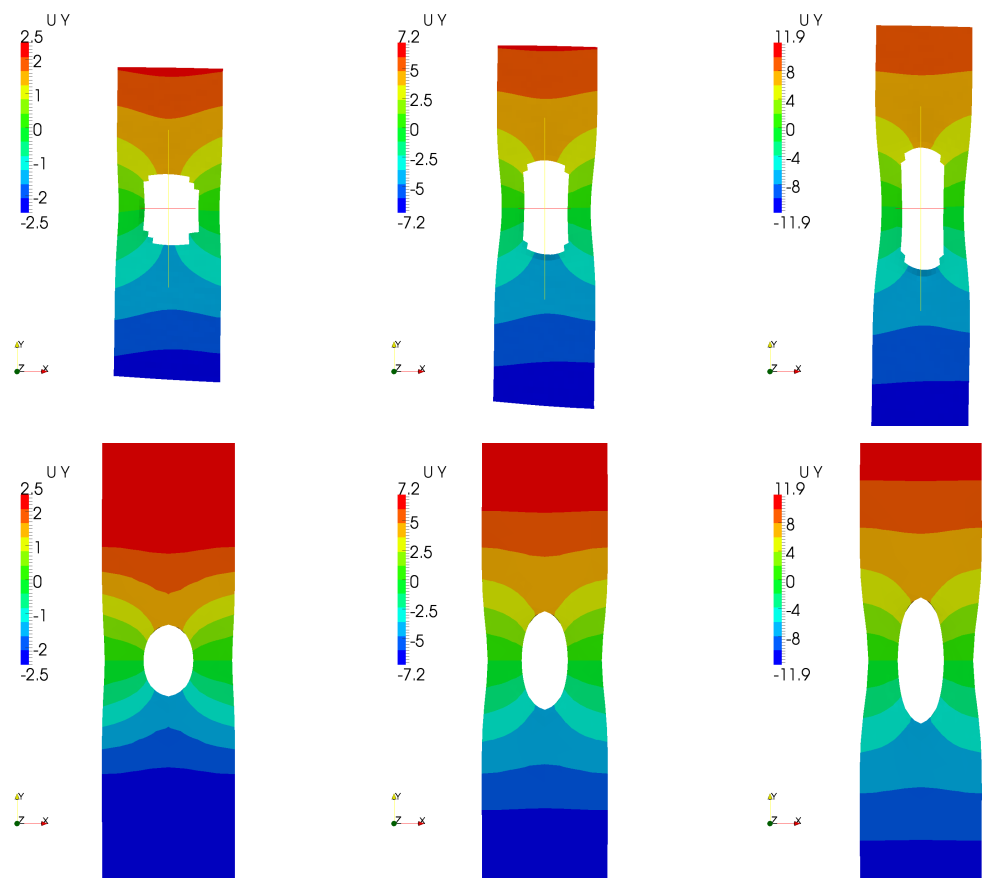

Figure 12. Perforated strip: contour plot of displacement in vertical direction. Top: experiments. Bottom: simulations. Left: NLST 6. Center: NLST 18. Right: NLST 30.

with the values according to Table 2 . In this way it is possible to incorporate the measured force into the parameter identification and make it more robust with respect to nonsymmetric fixation in longitudinal direction. The following leastsquares functional is introduced:

$$
q_{\mathrm{LS}}(\underline{s})=Q(\boldsymbol{u}(\underline{s}))=\sum_{i=1}^{n_{\text {tdat }}} \sum_{j=1}^{n_{\mathrm{mp}}}\left\|\underline{\boldsymbol{u}}_{i j}(\underline{s})-\underline{\overline{\boldsymbol{u}}}_{i j}\right\| .
$$

Here $\overline{\boldsymbol{u}}_{i j}=\left[\bar{u}_{x}, \bar{u}_{y}\right]_{i j}$ assembles experimental data for displacements at $n_{\mathrm{mp}}$ observation points for $n_{\text {tdat }}$ observation states, and $\underline{\boldsymbol{u}}_{i j}=\left[u_{x}, u_{y}\right]_{i j}$ assembles the corresponding simulated data. The number of load steps is $N=15$, the number of observation states according to Table 2 is $n_{\text {tdat }}=5$, and there are $n_{\mathrm{mp}}=35$ observation points. In this way the functional (61) represents a special case of (17) with the quantity of interest for the model error. The resulting vector for the material parameters after optimization is

$$
\underline{s}^{*}=\left[\begin{array}{c}
c_{1} \\
m_{1}
\end{array}\right]=\left[\begin{array}{r}
8.0997 \\
-1.9360
\end{array}\right] \text {. }
$$



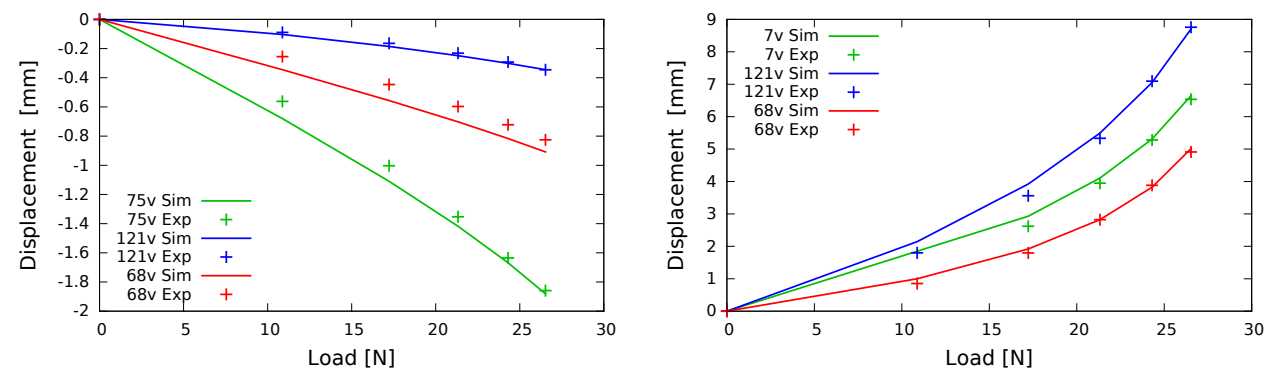

Figure 13. Perforated strip: displacements for experiment and simulation at different observation points. Left: horizontal displacement $u_{x}$ versus loading. Right: vertical displacement $u_{y}$ versus loading.
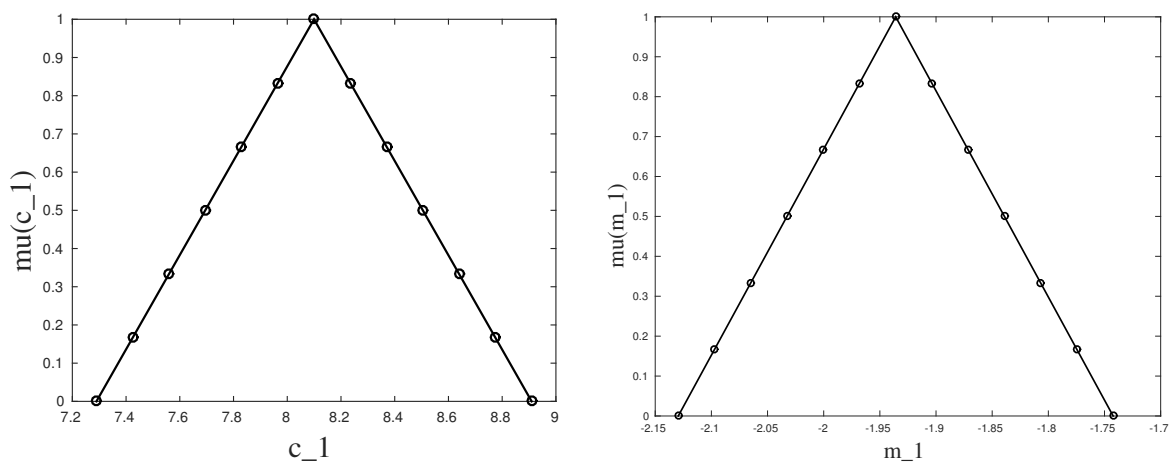

Figure 14. Perforated strip: input membership functions for material parameters. Left: parameter $c_{1}$. Right: parameter $m_{1}$.

For three of these steps, NLST $=6,18,30$, the measured displacement in the vertical direction on the surface of the specimen is presented in the first row of Figure 12. Please note that the colored area does not cover the whole surface of the specimen due to limitations of the measurement technology. However, the agreement to the results of simulation in the second row of Figure 12 is well established.

For some observation nodes the horizontal as well as the vertical displacements $u_{x}$ and $u_{y}$ are plotted over the load in Figure 13, left and right, respectively. The dots are the measured values, and the lines are the computed values. A very reasonable agreement is obtained for both types of quantities.

Based on the above results of parameter identification the following considerations are directed to the fuzzy analysis of the material parameters obtained in (62). To this end, firstly membership functions are generated according to Figure 14, as input quantities for the fuzzy analysis. The parameters in (60) are chosen as mean 

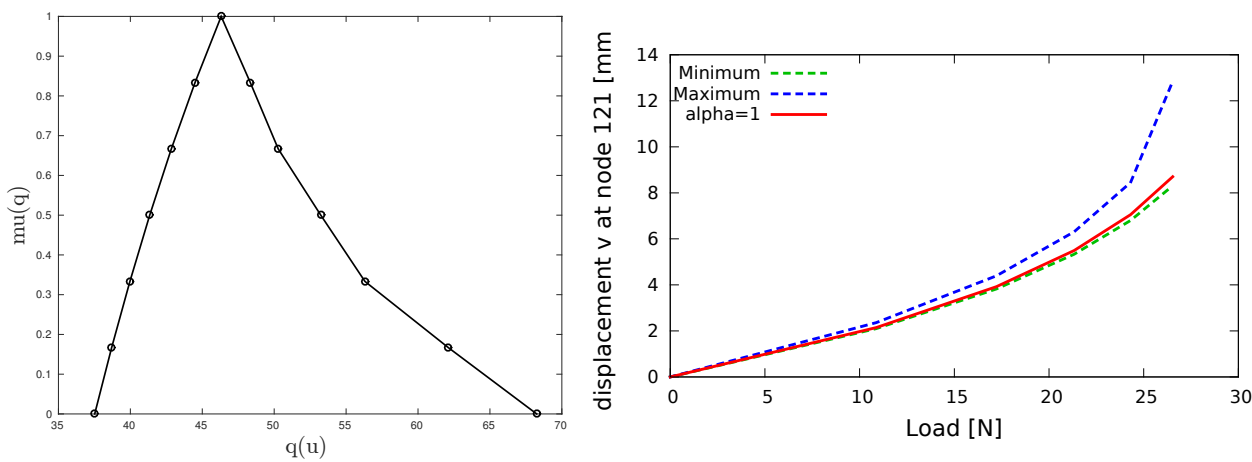

Figure 15. Perforated strip: fuzzy results for quantity of interest with displacements. Left: output membership function. Right: uncertainty regime for vertical displacement at observation point 121.

values to obtain triangular fuzzy numbers according to (44) as

$$
\hat{A}_{i}=\left\langle s_{i}^{L}, s_{i}^{M}, s_{i}^{R}\right\rangle=\left\langle 0.9 s_{i}^{*}, s_{i}^{*}, 1.1 s_{i}^{*}\right\rangle
$$

that is, for each material parameter a maximum deviation of $10 \%$ for the uncertainty is assumed. The $\alpha$-discretization is performed with 7 equally spaced intervals. In the fuzzy analysis we are firstly interested in the uncertainty of the quantity of interest

$$
q_{u}(\underline{s}):=Q(\boldsymbol{u}(\underline{s}))=\sum_{i=1}^{n_{\text {tdat }}} \sum_{j=1}^{n_{\mathrm{mp}}}\left\|\underline{\boldsymbol{u}}_{i j}(\underline{s})\right\|,
$$

with displacements $\underline{\boldsymbol{u}}_{i j}(\underline{s})$ according to (61). As a second quantity we choose the least-squares functional $q_{\mathrm{LS}}(\underline{s})$ in (61). For both quantities of interest the minimization and maximization problems P1a and P1b, respectively, according to (25) are solved at each $\alpha$-level.

Figure 15, left, shows the output membership functions $\mu\left(q_{u}\right)$ for the quantity of interest with displacements in (64). The corresponding Figure 15, right, displays the uncertainty regime for the vertical displacement $v$ at a specific observation point 121 displayed in Figure 11.

Figure 16 summarizes the fuzzy results for the quantity of interest formulated as a model error based on the least-squares functional in (64). Figure 16, left, shows the output membership functions $\mu_{\mathrm{LS}}\left(q_{\mathrm{LS}}\right)$. As explained in Section 4.4, the constraints (23) are not activated at the $\alpha$-levels, thus resulting in a vertical line in the left-hand side of the membership function in Figure 16, left. On the other hand, the maximization values $\max _{s \in \mathcal{S}_{\alpha_{k}}} q(s)$ exhibit a strong nonlinear behavior in Figure 16, left, influenced by the constraints (23) at the corresponding $\alpha$-level. 

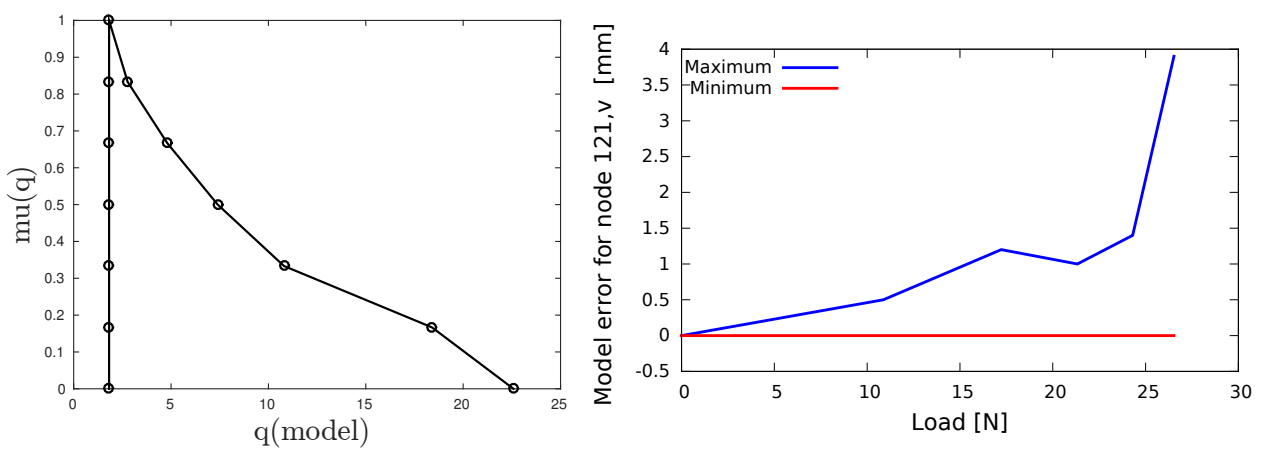

Figure 16. Perforated strip: fuzzy results for quantity of interest with model error. Left: output membership function. Right: uncertainty regime for model error at observation point 121 .

Figure 16, right, displays the related uncertainty regimes for the model errors of the displacement at the observation point 121.

The maximum deviations from the quantities at $\alpha=1$ for all three quantities are

$$
\begin{array}{cc}
\Delta_{u}^{L}=\frac{q_{u \alpha=0}^{L}-q_{u \alpha=1}^{L}}{q_{u \alpha=1}^{L}} 100=19.00 \%, & \Delta_{u}^{R}=\frac{q_{u \alpha=0}^{R}-q_{u \alpha=1}^{L}}{q_{u \alpha=1}^{L}} 100=47.36 \%, \\
\Delta_{\mathrm{ME}}^{L}=\frac{q_{\mathrm{LS} \alpha=0}^{L}-q_{\mathrm{LS} \alpha=1}^{L}}{q_{\mathrm{LS} \alpha=1}^{L}} 100=0 \%, & \Delta_{\mathrm{ME}}^{R}=\frac{q_{\mathrm{LS} \alpha=0}^{R}-q_{\mathrm{LS} \alpha=1}^{L}}{q_{\mathrm{LS} \alpha=1}^{L}} 100=1142 \%,
\end{array}
$$

thus revealing a comparatively large uncertainty especially for the model error.

\section{Conclusion}

This work is focused on epistemic uncertainties in the framework of continuum mechanics, which is taken into account with fuzzy analysis. The underlying min-max optimization problem of the extension principle is approximated by $\alpha$-discretization, resulting in a separation of minimum and maximum problems. To become more general, so-called quantities of interest are employed, which allow a general formulation for the target problem of interest.

Variational formulations have at least two intrinsic advantages.

- They enable a general framework for the analytical sensitivity analysis, which allows the effective solution of the related optimization problems with gradientbased methods.

- They give the basis for adaptive refinement of the underlying FE discretization. 
The relation to parameter identification problems based on least-squares functions is highlighted. In particular we have shown that the solution of the leastsquares problem coincides with the solution of the related minimization problems at all $\alpha$-levels.

The solutions of the related optimization problems with simple constraints are obtained with a gradient-based scheme, which is derived from a sensitivity analysis for the target problem. Two numerical examples for the fuzzy analysis of material parameters are concerned with a necking problem at large strain elastoplasticity and a perforated strip at large strain hyperelasticity. Further research will be directed to the following topics.

- Future investigations could be focused on different kinds of design variables, in addition to material parameters considered in the examples of this work.

- The adoption of adaptive mesh refinement which is driven by a goal quantity of interest results in more accurate results at fewer degrees of freedom and therefore is of great importance [Widany and Mahnken 2012].

- In most simulations uncertainties of different types occur. Therefore, the type of epistemic uncertainty should be combined with the type of aleatoric uncertainty in a polymorphic uncertainty model; see, e.g., [Graf et al. 2015].

- The present article is focused on the case of simple constraints. Therefore, the interaction of the fuzzy variables is a further challenge in future work on fuzzy analysis [Möller and Beer 2004].

\section{Appendix A: Second-order sensitivity analysis}

This appendix provides the second-order sensitivity analysis complementary to the first-order sensitivity analysis in Section 4.

A.1. The direct differentiation method. We begin the second-order analysis by the direct differentiation method analogously to the first-order analysis for derivation of (29). To this end, we apply the chain rule to the reduced functionals in (29a) and $(29 b)$ :

$$
\begin{aligned}
g^{\prime \prime}\left(\underline{s} ; \delta \underline{s}_{1}, \delta \underline{s}_{2}\right)= & G_{\underline{s}}^{\prime \prime}\left(\underline{s}, \boldsymbol{u}(\underline{s}) ; \delta \boldsymbol{u}, \delta \underline{s}_{1}, \delta \underline{s}_{2}\right)+G_{\boldsymbol{u} \underline{s}}^{\prime \prime}\left(\underline{s}, \boldsymbol{u}(\underline{s}) ; \delta \boldsymbol{u}, \bar{\delta} \boldsymbol{u}_{1}, \delta \underline{s}_{2}\right) \\
& +G_{\underline{s} \boldsymbol{u}}^{\prime \prime}\left(\underline{s}, \boldsymbol{u}(\underline{s}) ; \delta \boldsymbol{u}, \delta \underline{s}_{1}, \bar{\delta} \boldsymbol{u}_{2}\right)+G_{\boldsymbol{u} \boldsymbol{u}}^{\prime \prime}\left(\underline{s}, \boldsymbol{u}(\underline{s}) ; \delta \boldsymbol{u}, \bar{\delta} \boldsymbol{u}_{1}, \bar{\delta} \boldsymbol{u}_{2}\right) \\
& +G_{\boldsymbol{u}}^{\prime}\left(\underline{s}, \boldsymbol{u}(\underline{s}) ; \delta \boldsymbol{u}, \bar{\delta}^{2} \boldsymbol{u}\right)=0 \\
& \quad \text { for all } \delta \boldsymbol{u} \in \mathscr{U}^{0} \text { and } \delta \underline{s}_{1}, \delta \underline{s}_{2} \in \mathcal{S}_{\alpha_{k}}, \quad(A) \\
q^{\prime \prime}\left(\underline{s} ; \delta \underline{s}_{1}, \delta \underline{s}_{2}\right)= & Q_{\underline{s} \underline{s}}^{\prime \prime}\left(\underline{s}, \boldsymbol{u}(\underline{s}) ; \delta \underline{s}_{1}, \delta \underline{s}_{2}\right)+Q_{\boldsymbol{u} \underline{s}}^{\prime \prime}\left(\underline{s}, \boldsymbol{u}(\underline{s}) ; \bar{\delta} \boldsymbol{u}_{1}, \delta \underline{s}_{2}\right) \\
& +Q_{\underline{s} \boldsymbol{u}}^{\prime \prime}\left(\underline{s}, \boldsymbol{u}(\underline{s}) ; \delta \underline{s}_{1}, \bar{\delta} \boldsymbol{u}_{2}\right)+Q_{u \boldsymbol{u}}^{\prime \prime}\left(\underline{s}, \boldsymbol{u}(\underline{s}) ; \bar{\delta} \boldsymbol{u}_{1}, \bar{\delta} \boldsymbol{u}_{2}\right) \\
& +Q_{u}^{\prime}\left(\underline{s}, \boldsymbol{u}(\underline{s}) ; \bar{\delta}^{2} \boldsymbol{u}\right) \text { for all } \delta \underline{s}_{1}, \delta \underline{s}_{2} \in \mathcal{S}_{\alpha_{k}},
\end{aligned}
$$


where analogously to (29) $G_{s s}^{\prime \prime}(\cdot, \cdot ; \cdot ; \cdot), G_{u s}^{\prime \prime}(\cdot, \cdot ; \cdot, \cdot, \cdot)$, etc., denote partial derivatives with respect to $\underline{s}$ and $\boldsymbol{u}$, defined as Gâteaux derivatives. Equation (A-1a) is zero since $\boldsymbol{u}(\underline{s})$ solves the tangent problem (29a) for each $\underline{s} \in \mathcal{S}$ [Johansson et al. 2007; Mahnken and Stein 1996] in the context of homogeneous deformation problems. Equation (A-1b) follows by differentiation of (29b). Inserting the solution $\bar{\delta}^{2} \boldsymbol{u}:=\boldsymbol{u}_{s s}^{\prime \prime}\left(\underline{s} ; \delta \underline{s}_{1}, \delta \underline{s}_{2}\right)$ of (A-1a) into (A-1b) may be used to evaluate $q^{\prime \prime}\left(\underline{s} ; \delta \underline{s}_{1}, \delta \underline{s}_{2}\right)$. With this result for $q^{\prime \prime}$ we introduce the Hessian of the quantity of interest

$$
\underline{H}=\nabla^{2} q(\underline{s}) \in \mathcal{S} \times \mathcal{S}, \quad \text { where }\left(\nabla^{2} q(\underline{s})\right)_{i j}=q^{\prime \prime}\left(\underline{s} ; \underline{e}_{i}, \underline{e}_{j}\right), i, j=1, \ldots, n_{s} .
$$

Then, for the case of simple constraints in (24), the sufficient condition (27b) simplifies in terms of a reduced Hessian $\underline{\bar{H}}$ as [Bertsekas 1982]

$$
\delta \underline{s}^{T} \underline{\bar{H}} \delta \underline{s} \geq \gamma\|\delta \underline{s}\|^{2} \quad \text { for all } \bar{\delta} \underline{s} \in \mathbb{R}^{n_{s}-n_{B}},
$$

$$
\text { where } \begin{aligned}
\bar{H}_{I J} & =\left\{\nabla^{2} q(\underline{s})\right\}_{I J}=\frac{\partial^{2} q}{\partial s_{I} \partial s_{J}}, \quad I, J \notin B(\underline{s}), \\
B(\underline{s}) & =\left\{i \mid s_{i}=\left(s_{i, \alpha_{k}}^{L}, \frac{\partial q}{\partial s_{i}} \geq 0\right) \vee s_{i}=\left(s_{i, \alpha_{k}}^{R}, \frac{\partial q}{\partial s_{i}} \leq 0\right)\right\},
\end{aligned}
$$

where $B(\underline{s})$ denotes the set of indices of binding constraints at $\underline{s}$ in accordance with the necessary condition (31) and $n_{B}=\operatorname{dim}\{B(\underline{s})\}$ is its dimension.

A.2. The adjoint state differentiation method. Alternatively to the direct differentiation method in Appendix A.1 the second derivative of the quantity of interest $q^{\prime \prime}\left(\underline{s} ; \delta \underline{s}_{1}, \delta \underline{s}_{2}\right)$ is obtained by exploiting the adjoint equation (36b) analogously to the first-order analysis for derivation of the equations (37). Setting $\delta \boldsymbol{u}=\bar{\delta}^{2} \boldsymbol{u}$ in (36b) and using the result (A-1a) with the choice $\delta \boldsymbol{u}=\boldsymbol{\Lambda}$, we obtain

$$
\begin{array}{r}
Q_{u}^{\prime}\left(\underline{s}, \boldsymbol{u} ; \bar{\delta}^{2} \boldsymbol{u}\right)=-G_{\boldsymbol{u}}^{\prime}\left(\underline{s}, \boldsymbol{u} ; \boldsymbol{\Lambda}, \bar{\delta}^{2} \boldsymbol{u}\right) \\
=G_{\underline{s} \underline{\underline{s}}}^{\prime \prime}\left(\underline{s}, \boldsymbol{u} ; \boldsymbol{\Lambda}, \delta \underline{s}_{1}, \delta \underline{s}_{2}\right)+G_{\boldsymbol{u} \underline{\underline{s}}}^{\prime \prime}\left(\underline{s}, \boldsymbol{u} ; \boldsymbol{\Lambda}, \bar{\delta} \boldsymbol{u}_{1}, \delta \underline{s}_{2}\right) \\
\quad+G_{\underline{s} \boldsymbol{u}}^{\prime \prime}\left(\underline{s}, \boldsymbol{u} ; \boldsymbol{\Lambda}, \delta \underline{s}_{1}, \bar{\delta} \boldsymbol{u}_{2}\right)+G_{\boldsymbol{u} \boldsymbol{u}}^{\prime \prime}\left(\underline{s}, \boldsymbol{u} ; \boldsymbol{\Lambda}, \bar{\delta} \boldsymbol{u}_{1}, \bar{\delta} \boldsymbol{u}_{2}\right) \\
\quad \text { for all } \delta \underline{s}_{1}, \delta \underline{s}_{2} \in \mathcal{S}_{\alpha_{k}},
\end{array}
$$

where $\bar{\delta} \boldsymbol{u}_{k}=\boldsymbol{u}_{s}^{\prime}\left(\underline{s} ; \delta \underline{s}_{k}\right), k=1,2$, and where it is noteworthy to recall the relation $\left.\bar{\delta}^{2} \boldsymbol{u}=\boldsymbol{u}_{\underline{s}}^{\prime \prime} \underline{\underline{s}} ; \delta \underline{s}_{1}, \delta \underline{s}_{2}\right)$. Inserting the result (A-4) into (A-1b), the required result for the second derivative of the quantity of interest by the adjoint state method 
becomes

$$
\begin{aligned}
q^{\prime \prime}\left(\underline{s} ; \delta \underline{s}_{1}, \delta \underline{s}_{2}\right)=Q_{\underline{s} \underline{s}}^{\prime \prime} & \left(\underline{s}, \boldsymbol{u} ; \delta \underline{s}_{1}, \delta \underline{s}_{2}\right)+Q_{\underline{u} \underline{\underline{s}}}^{\prime \prime}\left(\underline{s}, \boldsymbol{u} ; \bar{\delta} \boldsymbol{u}_{1}, \delta \underline{s}_{2}\right) \\
& +Q_{\underline{s} \boldsymbol{u}}^{\prime \prime}\left(\underline{s}, \boldsymbol{u} ; \delta \underline{s}_{1}, \bar{\delta} \boldsymbol{u}_{2}\right)+Q_{\boldsymbol{u} \boldsymbol{u}}^{\prime \prime}\left(\underline{s}, \boldsymbol{u} ; \bar{\delta} \boldsymbol{u}_{1}, \bar{\delta} \boldsymbol{u}_{2}\right) \\
& +G_{\underline{s} \underline{s}}^{\prime \prime}\left(\underline{s}, \boldsymbol{u} ; \boldsymbol{\Lambda}, \delta \underline{s}_{1}, \delta \underline{s}_{2}\right)+G_{\boldsymbol{u} \underline{\underline{s}}}^{\prime \prime}\left(\underline{s}, \boldsymbol{u} ; \boldsymbol{\Lambda}, \bar{\delta} \boldsymbol{u}_{1}, \delta \underline{s}_{2}\right) \\
& +G_{\underline{s} \boldsymbol{u}}^{\prime \prime}\left(\underline{s}, \boldsymbol{u} ; \boldsymbol{\Lambda}, \delta \underline{s}_{1}, \bar{\delta} \boldsymbol{u}_{2}\right)+G_{\boldsymbol{u} \boldsymbol{u}}^{\prime \prime}\left(\underline{s}, \boldsymbol{u} ; \boldsymbol{\Lambda}, \bar{\delta} \boldsymbol{u}_{1}, \bar{\delta} \boldsymbol{u}_{2}\right) \\
& \quad \text { for all } \delta \underline{s}_{1}, \delta \underline{s}_{2} \in \mathcal{S}_{\alpha_{k}} ;
\end{aligned}
$$

compare this to [Vexler 2004].

\section{Appendix B: Sensitivity analysis for large strain formulations}

This appendix provides an overall summary for determination of the state problem (6) as a variational formulation, the generally unsymmetric tangent bilinear form (8) of the Newton method for FEM equilibrium iteration, and the gradient $\nabla_{s} f$ for the projection algorithm (47), provided the underlying model equations are formulated within a large strain framework. More details and related references are documented, e.g., in [Barthold 1993; Mahnken 2004; Mahnken and Stein 1997].

B.1. Time and design (tangent) derivatives of kinematic variables. From the fundamental mapping in (3) the design derivative is

$$
\boldsymbol{\varphi}_{\underline{s}}^{\prime}(\boldsymbol{X}, t, \underline{s} ; \delta \underline{s})=\left(\partial_{X} \boldsymbol{\varphi}(\boldsymbol{X}, t, \underline{s}) \cdot \partial_{\underline{s}} \boldsymbol{X}+\partial_{t} \boldsymbol{\varphi}(\boldsymbol{X}, t, \underline{s}) \cdot \partial_{\underline{s}} t+\partial_{\underline{s}}^{p} \boldsymbol{\varphi}(\boldsymbol{X}, t, \underline{s})\right) \cdot \delta \underline{s},
$$

where $\partial_{X} \varphi(X, t, \underline{s}) \in \mathbb{R}^{n_{\mathrm{dim}}} \times \mathbb{R}^{n_{\mathrm{dim}}}$ and $\partial_{t} \boldsymbol{\varphi} \in \mathbb{R}^{n_{\mathrm{dim}}} \times \mathcal{T}$ are partial tangents in the direction of $\boldsymbol{X}$ and $t$, respectively, and the notation

$$
\partial_{\underline{s}}^{p}\{\cdot(\boldsymbol{\varphi}(\boldsymbol{X}, t, \underline{s}), \underline{s})\}:=\partial_{\underline{s}}\{\cdot(\underline{s})\}
$$

is used, thus taking into account explicit dependence of the design variables.

It follows that formulations associated with the time derivative of a kinematic quantity carry over to the design derivative with minor modifications. To give an example, we consider the right Cauchy-Green tensor $\boldsymbol{C}=\boldsymbol{F}^{T} \boldsymbol{F}$ with the deformation gradient in (4a). Then the time and design tangents of $\boldsymbol{C}$ are

$$
\partial_{t} \boldsymbol{C}=2 \operatorname{sym}(\boldsymbol{C L})=: 2 \boldsymbol{D}, \quad \partial_{\underline{s}} \boldsymbol{C}=2 \operatorname{sym}\left(\boldsymbol{C} \boldsymbol{L}_{\underline{s}}\right)=: 2 \boldsymbol{D}_{\underline{s}} .
$$

According to Table B.1, $\boldsymbol{L}$ and $\boldsymbol{L}_{s}$ are material velocity gradients and $\boldsymbol{D}$ and $\boldsymbol{D}_{s}$ are material rate of deformation tensors with respect to the reference configuration. Some further examples of the analogy for time and design derivatives are also listed in Table B.1. Here $\boldsymbol{l}_{t}$ and $\boldsymbol{l}_{\underline{s}}$ are spatial velocity gradients induced by the velocities $\boldsymbol{v}_{t}$ and $\boldsymbol{v}_{\underline{s}}$, respectively, and $\boldsymbol{d}_{t}$ and $\boldsymbol{d}_{\underline{s}}$ are the associated spatial rate of deformation tensors [Barthold 1993; Mahnken 2004]. Also, the symmetric operator $\operatorname{sym}(\cdot):=$ $\frac{1}{2}\left((\cdot)^{T}+(\cdot)\right)$ and the covariant metric tensor $\boldsymbol{g}^{\mathrm{b}}$ are used. $\mathcal{L}_{t}$ denotes the Lie 


\begin{tabular}{|c|c|c|c|}
\hline$\dot{\boldsymbol{F}}=\nabla_{\boldsymbol{X}} \partial_{t} \boldsymbol{\varphi}$ & $\partial_{\underline{S}} \boldsymbol{F}=\nabla_{\boldsymbol{X}} \boldsymbol{\varphi}_{\underline{S}}^{\prime}$ & $\partial_{\delta} \boldsymbol{F}=\nabla_{\boldsymbol{X}} \delta \boldsymbol{u}$ & $\partial_{\Delta} \boldsymbol{F}=\nabla_{\boldsymbol{X}} \Delta \boldsymbol{u}$ \\
\hline $\boldsymbol{L}=\boldsymbol{F}^{-1} \dot{\boldsymbol{F}}$ & $\boldsymbol{L}_{\underline{S}}=\boldsymbol{F}^{-1} \partial_{\underline{S}} \boldsymbol{F}$ & $\boldsymbol{L}_{\delta}=\boldsymbol{F}^{-1} \partial_{\delta} \boldsymbol{F}$ & $\boldsymbol{L}_{\Delta}=\boldsymbol{F}^{-1} \partial_{\Delta} \boldsymbol{F}$ \\
\hline $\boldsymbol{D}=\operatorname{sym}(\boldsymbol{C} \boldsymbol{L})$ & $\boldsymbol{D}_{\underline{S}}=\operatorname{sym}\left(\boldsymbol{C} \boldsymbol{L}_{\underline{S}}\right)$ & $\boldsymbol{D}_{\delta}=\operatorname{sym}\left(\boldsymbol{C} \boldsymbol{L}_{\delta}\right)$ & $\boldsymbol{D}_{\Delta}=\operatorname{sym}\left(\boldsymbol{C} \boldsymbol{L}_{\Delta}\right)$ \\
\hline $\boldsymbol{l}=\dot{\boldsymbol{F}} \boldsymbol{F}^{-1}$ & $\boldsymbol{l}_{\underline{S}}=\partial_{\underline{S}} \boldsymbol{F} \boldsymbol{F}^{-1}$ & $\boldsymbol{l}_{\delta}=\partial_{\delta} \boldsymbol{F} \boldsymbol{F}^{-1}$ & $\boldsymbol{l}_{\Delta}=\partial_{\Delta} \boldsymbol{F} \boldsymbol{F}^{-1}$ \\
\hline $\boldsymbol{d}=\operatorname{sym}\left(\boldsymbol{g}^{\mathrm{b}} \boldsymbol{l}\right)$ & $\boldsymbol{d}_{\underline{S}}=\operatorname{sym}\left(\boldsymbol{g}^{\mathrm{b}} \boldsymbol{l}_{\underline{S}}\right)$ & $\boldsymbol{d}_{\delta}=\operatorname{sym}\left(\boldsymbol{g}^{\mathrm{b}} \boldsymbol{l}_{\delta}\right)$ & $\boldsymbol{d}_{\Delta}=\operatorname{sym}\left(\boldsymbol{g}^{\mathrm{b}} \boldsymbol{l}_{\Delta}\right)$ \\
\hline $\mathcal{L}_{t}(\cdot)=\Phi_{*}\left[\partial_{t} \Phi^{*}(\cdot)\right]$ & $\mathcal{L}_{\underline{S}}(\cdot)=\Phi_{*}\left[\partial_{\underline{S}} \Phi^{*}(\cdot)\right]$ & $\mathcal{L}_{\delta}(\cdot)=\Phi_{*}\left[\partial_{\delta} \Phi^{*}(\cdot)\right]$ & $\mathcal{L}_{\Delta}(\cdot)=\Phi_{*}\left[\partial_{\Delta} \Phi^{*}(\cdot)\right]$ \\
\hline
\end{tabular}

Table B.1. Examples for kinematic variables induced by time derivatives, design variation, virtual variation, and linearization increment.

(time) derivative of a spatial argument, with related pull-back $\Phi^{*}$ and push-forward operators $\Phi_{*}$.

For the variational formulation in Section 2, two additional types of quantities are useful: the virtual displacements $\delta \boldsymbol{u}$ with $\left.\delta \boldsymbol{u}\right|_{\partial_{\varphi} \mathcal{B}}=\boldsymbol{\Gamma}$ and the increments for linearization $\Delta \boldsymbol{u}$ with $\left.\Delta \boldsymbol{u}\right|_{\partial_{\varphi} \mathcal{B}}=\boldsymbol{\Gamma}$, both independent of $(t, \underline{s})$. With these quantities additional kinematical variables are defined in Table B.1, induced by $\delta \boldsymbol{u}$ or $\Delta \boldsymbol{u}$, respectively, in complete analogy to $\partial_{t} \varphi$ and $\varphi_{s}^{\prime}$, respectively. In Table B.1, ${ }^{*} \Phi_{e}^{\sharp}$ and ${ }_{*} \Phi_{e}^{\sharp}$ are pull-back and push-forward operators of a contravariant tensor according to (B-4) [Mahnken 2005]

$$
{ }^{*} \Phi_{e}^{\sharp}\left[\overline{\boldsymbol{a}}^{\sharp}\right]=\boldsymbol{F}_{\mathrm{el}}^{-1} \cdot \overline{\boldsymbol{a}}^{\sharp} \cdot \boldsymbol{F}_{\mathrm{el}}^{-t}, \quad * \Phi_{e}^{\sharp}\left[\overline{\boldsymbol{A}}^{\sharp}\right]=\boldsymbol{F}_{\mathrm{el}} \cdot \overline{\boldsymbol{A}}^{\sharp} \cdot \boldsymbol{F}_{\mathrm{el}}^{t} \cdot
$$

B.2. Time and design (tangent) derivatives of stress tensors. For alleviation of notation we shall restrict ourselves to large strain elasticity with homogeneous material governed by the local constitutive equations for the second Piola-Kirchhoff stress tensor $\boldsymbol{T}=J \boldsymbol{F}^{-1} \boldsymbol{\sigma} \boldsymbol{F}^{-T}$ dependent on the right Cauchy-Green strain tensor $\boldsymbol{C}$. We remark that the results obtained below also hold for general constitutive models which incorporate inelastic behavior. Then, due to the functional relationship $\boldsymbol{T}=\hat{\boldsymbol{T}}(\hat{\boldsymbol{C}}(\boldsymbol{\varphi}(\boldsymbol{X}, t, \underline{s})), \underline{s})$ the dependence of $\hat{\boldsymbol{T}}$ with respect to time $t$ is implicit, while the dependence on the design variables $\underline{s}$ is both explicit and implicit. Consequently the time and design tangents of $\boldsymbol{T}$ are

$$
\partial_{t} \boldsymbol{T}=\mathbb{C}: \boldsymbol{D}_{t}, \quad \partial_{\underline{s}} \boldsymbol{T}=\mathbb{C}: \boldsymbol{D}_{\underline{s}}+\partial_{\underline{s}}^{p} \boldsymbol{T},
$$

where $\mathbb{C}:=2 \partial_{\boldsymbol{C}} \boldsymbol{T}$ is the symmetric fourth-order material operator, and where the material deformation rate tensors $\boldsymbol{D}_{t}$ and $\boldsymbol{D}_{s}$ are defined in Table B.1. Here the notation (B-2) is used, thus taking into account explicit dependence of the design variables. Comparatively, the time tangent of $\boldsymbol{T}$ consists of one part, while the design tangent consists of two parts. 


\section{Material formulation}

- State equation as weak form

$G=\left\langle\boldsymbol{T}: \boldsymbol{D}_{\delta}\right\rangle-\bar{l}=0$

- Tangent bilinear form for Newton method

$G_{\boldsymbol{u}}^{\prime}=\left\langle\left(\mathbb{C}: \boldsymbol{D}_{\Delta}\right): \boldsymbol{D}_{\delta}+\boldsymbol{L}_{\Delta} \boldsymbol{T}: \boldsymbol{D}_{\delta}\right\rangle$

- Tangent problem for parameter sensitivity $g^{\prime}(\underline{s} ; \delta \underline{s})=\left\langle\left(\mathbb{C}: \boldsymbol{D}_{\underline{s}}\right): \boldsymbol{D}_{\delta}+\boldsymbol{L}_{\underline{s}} \boldsymbol{T}: \boldsymbol{D}_{\delta}+\partial_{\underline{s}}^{p} \boldsymbol{T}: \boldsymbol{D}_{\delta}\right\rangle$ $=0$

$$
\begin{aligned}
& \text { Spatial formulation } \\
& G=\left\langle\boldsymbol{\tau}: \boldsymbol{d}_{\delta}\right\rangle-\bar{l}=0 \\
& G_{\boldsymbol{u}}^{\prime}=\left\langle\left(\mathbb{c}: d_{\Delta}\right): d_{\delta}+l_{\Delta} \tau: d_{\delta}\right\rangle \\
& g^{\prime}(\underline{s} ; \delta \underline{s})=\left\langle\left(\mathbb{C}: \boldsymbol{d}_{\underline{s}}\right): \boldsymbol{d}_{\delta}+\boldsymbol{l}_{\underline{s}} \boldsymbol{\tau}: \boldsymbol{d}_{\delta}+\partial_{\underline{s}}^{p} \boldsymbol{\tau}: \boldsymbol{d}_{\delta}\right\rangle \\
& =0
\end{aligned}
$$

Table B.2. State equation, linearization, and linear equation for parameter sensitivity in a material and a spatial formulation.

For the Kirchhoff stress tensor, which is obtained by push-forward of the second Piola-Kirchhoff stress tensor $\tau=\Phi_{*}(\boldsymbol{T})$, the associated time and design tangents are

$$
\partial_{t} \boldsymbol{\tau}=\mathbb{C}: \boldsymbol{d}_{t}+2 \operatorname{sym}\left(\boldsymbol{l}_{t} \boldsymbol{\tau}\right), \quad \partial_{\underline{s}} \boldsymbol{\tau}=\mathbb{C}: \boldsymbol{d}_{\underline{s}}+2 \operatorname{sym}\left(\boldsymbol{l}_{\underline{s}} \boldsymbol{\tau}\right)+\partial_{\underline{s}}^{p} \boldsymbol{\tau}
$$

and where $\partial_{\underline{s}}^{p} \boldsymbol{\tau}:=\boldsymbol{F} \partial_{\underline{s}}^{p} \boldsymbol{T} \boldsymbol{F}^{T}$. Here the spatial tangent module $\mathbb{C}$ is obtained by pushing forward its material counterpart, i.e., $\mathbb{C}=\Phi_{*}(\mathbb{C})$. Comparing the results for the time and design derivatives of the kinematic variable $C$ in (B-3) with the results of (B-5) and (B-6) for the stress tensors, it is observed that in (B-3) the structure for both derivatives is identical whereas in (B-5) and (B-6) the structures are different.

B.3. Numerical solution of the direct and the inverse problem. With the notations of the previous subsection the direct problem (6) as a weak form of the momentum equation is given in a material setting and a spatial setting in Table B.2. Here the rate of deformation tensor $\boldsymbol{D}_{\delta}$ induced by the virtual displacement $\delta \boldsymbol{u}$ is defined analogously to $\boldsymbol{D}_{t}$ and $\boldsymbol{D}_{\underline{s}}$ in Table B.1, and $\bar{l}$ designates the external part of the weak form.

The iterative solution of the state problem (6) is based on a Newton method (8) with tangent bilinear form $G_{\boldsymbol{u}}^{\prime}=G_{\boldsymbol{u}}^{\prime}(\underline{s}, \tilde{\boldsymbol{u}}(\underline{s}) ; \delta \boldsymbol{u}, \Delta \boldsymbol{u})$ summarized in Table B.2, both in a material and a spatial setting. The rate of deformation tensor $\boldsymbol{D}_{\Delta}$ induced by the incremental configuration $\Delta \varphi$ is defined in complete analogy to $\boldsymbol{D}_{t}$ and $\boldsymbol{D}_{\underline{s}}$ in Table B.1. The iterative solution of the minimization problem (45) is based on the iteration scheme (47). According to (29a) the tangent problem $g^{\prime}(\underline{s} ; \delta \underline{s})=$ $G_{s}^{\prime}(\underline{s}, \boldsymbol{u}(\underline{s}) ; \delta \boldsymbol{u}, \delta \underline{s})+G_{\boldsymbol{u}}^{\prime}(\underline{s}, \boldsymbol{u}(\underline{s}) ; \delta \boldsymbol{u}, \Delta \overline{\boldsymbol{u}})=0$, summarized in Table B.2 both in a material and a spatial setting, has to be solved at each iteration step for calculation of the sensitivity $\Delta \overline{\boldsymbol{u}}=\nabla \boldsymbol{u}(\underline{s}) \cdot \delta \underline{s}, \nabla \boldsymbol{u}(\underline{s}) \in \mathscr{U}^{0} \times \mathcal{S}$. As outlined extensively in [Mahnken and Stein 1997], solution of the tangent problem for history-dependent problems requires an additional postprocessing step for the history-dependent sensitivities. 


\section{References}

[Akpan et al. 2001] U. O. Akpan, T. S. Koko, I. R. Orisamolu, and B. K. Gallant, "Practical fuzzy finite element analysis of structures", Finite Elem. Anal. Des. 38:2 (2001), 93-111.

[Andresen et al. 1996] K. Andresen, S. Dannemeyer, H. Friebe, R. Mahnken, R. Ritter, and E. Stein, "Parameteridentifikation für ein plastisches Stoffgesetz mit FE-Methoden und Rasterverfahren", Bauingenieur 71 (1996), 21-31.

[Bangerth and Rannacher 2003] W. Bangerth and R. Rannacher, Adaptive finite element methods for differential equations, Birkhäuser, 2003.

[Banks and Kunisch 1989] H. T. Banks and K. Kunisch, Estimation techniques for distributed parameter systems, Systems \& Control: Foundations \& Applications 1, Birkhäuser, 1989.

[Barthold 1993] F.-J. Barthold, Theorie und Numerik zur Berechnung und Optimierung von Strukturen aus isotropen, hyperelastischen Materialien, Dr.-Ing. Dissertation, Universität Hannover, 1993.

[Bertsekas 1982] D. P. Bertsekas, "Projected Newton methods for optimization problems with simple constraints", SIAM J. Control Optim. 20:2 (1982), 221-246.

[Caylak and Mahnken 2012] I. Caylak and R. Mahnken, "Stabilization of mixed tetrahedral elements at large deformations", Internat. J. Numer. Meth. Eng. 90:2 (2012), 218-242.

[De Munck et al. 2008] M. De Munck, D. Moens, W. Desmet, and D. Vandepitte, “A response surface based optimisation algorithm for the calculation of fuzzy envelope FRFs of models with uncertain properties", Comput. Struct. 86:10 (2008), 1080-1092.

[Dennis and Schnabel 1983] J. E. Dennis, Jr. and R. B. Schnabel, Numerical methods for unconstrained optimization and nonlinear equations, Prentice Hall, 1983.

[Dubois and Prade 1980] D. Dubois and H. Prade, Fuzzy sets and systems: theory and applications, Mathematics in Science and Engineering 144, Academic, 1980.

[Farkas et al. 2008] L. Farkas, D. Moens, D. Vandepitte, and W. Desmet, "Application of fuzzy numerical techniques for product performance analysis in the conceptual and preliminary design stage”, Comput. Struct. 86:10 (2008), 1061-1079.

[Graf et al. 2015] W. Graf, M. Götz, and M. Kaliske, "Analysis of dynamical processes under consideration of polymorphic uncertainty”, Struct. Saf. 52:B (2015), 194-201.

[Johansson et al. 2007] H. Johansson, K. Runesson, and F. Larsson, "Parameter identification with sensitivity assessment and error computation”, GAMM-Mitt. 30:2 (2007), 430-457.

[Johansson et al. 2008] H. k. Johansson, F. Larsson, and K. Runesson, "Estimation of model errors in the calibration of viscoelastic material models", Internat. J. Numer. Meth. Eng. 76:10 (2008), $1568-1582$.

[Luenberger 1984] D. G. Luenberger, Linear and nonlinear programming, 2nd ed., Addison-Wesley, 1984.

[Mahnken 1993] R. Mahnken, Duale Verfahren für nichtlineare Optimierungsprobleme in der Strukturmechanik, Dr.-Ing. Dissertation, Universität Hannover, 1993.

[Mahnken 2004] R. Mahnken, "Identification of material parameters for constitutive equations", Chapter 19, pp. 637-655 in Encyclopedia of computational mechanics, vol. 2: Solids and structures, edited by E. Stein et al., Wiley, 2004.

[Mahnken 2005] R. Mahnken, "Void growth in finite deformation elasto-plasticity due to hydrostatic stress states", Comput. Meth. Appl. Mech. Eng. 194:34-35 (2005), 3689-3709.

[Mahnken 2013] R. Mahnken, "Goal-oriented adaptive refinement for phase field modeling with finite elements”, Internat. J. Numer. Meth. Eng. 94:4 (2013), 418-440. 
[Mahnken and Stein 1996] R. Mahnken and E. Stein, "Parameter identification for viscoplastic models based on analytical derivatives of a least-squares functional and stability investigations", Int. J. Plasticity 12:4 (1996), 451-479.

[Mahnken and Stein 1997] R. Mahnken and E. Stein, "Parameter identification for finite deformation elasto-plasticity in principal directions", Comput. Meth. Appl. Mech. Eng. 147:1-2 (1997), 17-39.

[Marsden and Hughes 1994] J. E. Marsden and T. J. R. Hughes, Mathematical foundations of elasticity, Dover, 1994.

[Möller and Beer 2004] B. Möller and M. Beer, Fuzzy randomness: uncertainty in civil engineering and computational mechanics, Springer, 2004.

[Möller et al. 2000] B. Möller, W. Graf, and M. Beer, "Fuzzy structural analysis using $\alpha$-level optimization", Comput. Mech. 26:6 (2000), 547-565.

[Muhanna and Mullen 1999] R. L. Muhanna and R. L. Mullen, "Formulation of fuzzy finite-element methods for solid mechanics problems", Comput.-Aided Civ. Inf. 14:2 (1999), 107-117.

[Nocedal and Wright 1999] J. Nocedal and S. J. Wright, Numerical optimization, Springer, 1999.

[Oden et al. 2006] J. T. Oden, S. Prudhomme, A. Romkes, and P. T. Bauman, "Multiscale modeling of physical phenomena: adaptive control of models", SIAM J. Sci. Comput. 28:6 (2006), 23592389.

[Ogden 1997] R. W. Ogden, Non-linear elastic deformations, Dover, 1997.

[Press et al. 1992] W. H. Press, S. A. Teukolsky, W. T. Vetterling, and B. P. Flannery, Numerical recipes in FORTRAN: the art of scientific computing, 2nd ed., Cambridge University, 1992.

[Quagliarella and Vicini 1998] D. Quagliarella and A. Vicini, "Coupling genetic algorithms and gradient based optimization techniques", Chapter 14, pp. 298-309 in Genetic algorithms and evolution strategy in engineering and computer science: recent advances and industrial applications, edited by D. Quagliarella et al., Wiley, 1998.

[Rüter 2004] M. Rüter, Error-controlled adaptive finite element methods in large strain hyperelasticity and fracture mechanics, Dr.-Ing. Dissertation, Universität Hannover, 2004.

[Schwefel 1977] H. P. Schwefel, Numerische Optimierung von Computer-Modellen mittels der Evolutionsstrategie: mit einer vergleichenden Einführung in die Hill-Climbing- und Zufallsstrategie, Interdisziplinäre Systemforschung 26, Birkhäuser, 1977.

[Simo and Armero 1992] J. C. Simo and F. Armero, "Geometrically nonlinear enhanced strain mixed methods and the method of incompatible modes", Internat. J. Numer. Meth. Eng. 33:7 (1992), 14131449 .

[Simo and Miehe 1992] J. C. Simo and C. Miehe, "Associative coupled thermoplasticity at finite strains: formulation, numerical analysis and implementation”, Comput. Meth. Appl. Mech. Eng. 98:1 (1992), 41-104.

[Simo and Taylor 1991] J. C. Simo and R. L. Taylor, "Quasi-incompressible finite elasticity in principal stretches: continuum basis and numerical algorithms", Comput. Meth. Appl. Mech. Eng. 85:3 (1991), 273-310.

[Sullivan 2015] T. J. Sullivan, Introduction to uncertainty quantification, Texts in Applied Mathematics 63, Springer, 2015.

[Vexler 2004] B. Vexler, Adaptive finite element methods for parameter identification problems, Dr. Dissertation, Universität Heidelberg, 2004.

[Viertl 1996] R. Viertl, Statistical methods for non-precise data, CRC, 1996.

[Wasfy and Noor 1998] T. Wasfy and A. Noor, "Application of fuzzy sets to transient analysis of space structures", pp. 1172-1182 in 39th AIAA/ASME/ASCE/AHS/ASC Structures, Structural Dynamics, and Materials Conference and Exhibit (Long Beach, CA, 1998), AIAA, 1998. 
[Widany and Mahnken 2012] K.-U. Widany and R. Mahnken, "Adaptivity for parameter identification of incompressible hyperelastic materials using stabilized tetrahedral elements", Comput. Meth. Appl. Mech. Eng. 245-246 (2012), 117-131.

[Zadeh 1965] L. A. Zadeh, "Fuzzy sets", Inform. Control 8 (1965), 338-353.

Received 17 Dec 2016. Accepted 27 Jun 2017.

ROLF MAHNKEN: rolf.mahnken@ltm.upb.de

Paderborn, Germany 
EDITORIAL BOARD

ANTONIO CARCATERRA

ERIC A. CARLEN

FRANCESCO DELL'ISOLA

RAFFAELE ESPOSITO

ALBERT FANNJIANG

Gilles A. FranCFORT

Pierangelo MARCATI

JEAN-JACQUES MARIGO

PETER A. MARKOWICH

MARTIN OSTOJA-STARZEWSKI

PIERRE SEPPECHER

DAVID J. STEIGMANN

PAUl STEINMANN

PierRe M. SuQueT

MANAGING EDITORS

MICOL AMAR

CORRADO LATTANZIO

ANGELA MADEO

MARTIN OSTOJA-STARZEWSKI

\section{ADVISORY BOARD}

ADNAN AKAY

Holm AltenBaCH

MICOL AMAR

HARM ASKES

TEODOR ATANACKOVIĆ

VICTOR BERDICHEVSKY

GUY BOUCHITTÉ

ANDREA BRAIDES

ROBERTO CAMASSA

MAURO CARFORE

ERIC DARVE

FELIX DARVE

ANNA DE MASI

GianPiEtro DEL Piero

EMMANUELE Di BENEDETTO

BERNOLD FIEDLER

IRENE M. GAMBA

DAVID Y. GAO

SERGEY GAVRILYUK

TIMOTHY J. HEALEY

DOMINIQUE JEULIN

ROGER E. KHAYAT

CORRADO LATTANZIO

ROBERT P. LIPTON

ANGELO LUONGO

ANGELA MADEO

JUAN J. MANFREDI

CARLO MARCHIORO

GÉRARD A. MAUGIN

ROBERTO NATALINI PATRIZIO NEFF

ANDREY PIATNITSKI

ERRICO PRESUTTI

MARIO PULVIRENTI

LUCIO RUSSO

Miguel A. F. SANJUAN

PATRICK SElvadurai

ALEXANDER P. SEYRANIAN

MIROSLAV ŠILHAVÝ

GUIDO SWEERS

ANTOINETTE TORDESILLAS

LEV TRUSKINOVSKY

JUAN J. L. VELÁZQUEZ VINCENZO VESPRI ANGELO VULPIANI msp.org/memocs

Università di Roma “La Sapienza”, Italia

Rutgers University, USA

(CO-CHAIR) Università di Roma "La Sapienza", Italia

(TREASURER) Università dell'Aquila, Italia

University of California at Davis, USA

(CO-CHAIR) Université Paris-Nord, France

Università dell'Aquila, Italy

École Polytechnique, France

DAMTP Cambridge, UK, and University of Vienna, Austria

(CHAIR MANAGING EDITOR) Univ. of Illinois at Urbana-Champaign, USA

Université du Sud Toulon-Var, France

University of California at Berkeley, USA

Universität Erlangen-Nürnberg, Germany

LMA CNRS Marseille, France

Università di Roma "La Sapienza", Italia

Università dell'Aquila, Italy

Université de Lyon-INSA (Institut National des Sciences Appliquées), France

(CHAIR MANAGING EDITOR) Univ. of Illinois at Urbana-Champaign, USA

Carnegie Mellon University, USA, and Bilkent University, Turkey

Otto-von-Guericke-Universität Magdeburg, Germany

Università di Roma "La Sapienza", Italia

University of Sheffield, UK

University of Novi Sad, Serbia

Wayne State University, USA

Université du Sud Toulon-Var, France

Università di Roma Tor Vergata, Italia

University of North Carolina at Chapel Hill, USA

Università di Pavia, Italia

Stanford University, USA

Institut Polytechnique de Grenoble, France

Università dell'Aquila, Italia

Università di Ferrara and International Research Center MEMOCS, Italia

Vanderbilt University, USA

Freie Universität Berlin, Germany

University of Texas at Austin, USA

Federation University and Australian National University, Australia

Université Aix-Marseille, France

Cornell University, USA

École des Mines, France

University of Western Ontario, Canada

Università dell' Aquila, Italy

Louisiana State University, USA

Università dell'Aquila, Italia

Université de Lyon-INSA (Institut National des Sciences Appliquées), France University of Pittsburgh, USA

Università di Roma "La Sapienza”, Italia

Université Paris VI, France

Istituto per le Applicazioni del Calcolo "M. Picone", Italy

Universität Duisburg-Essen, Germany

Narvik University College, Norway, Russia

Università di Roma Tor Vergata, Italy

Università di Roma "La Sapienza”, Italia

Università di Roma “Tor Vergata”, Italia

Universidad Rey Juan Carlos, Madrid, Spain

McGill University, Canada

Moscow State Lomonosov University, Russia

Academy of Sciences of the Czech Republic

Universität zu Köln, Germany

University of Melbourne, Australia

École Polytechnique, France

Bonn University, Germany

Università di Firenze, Italia

Università di Roma La Sapienza, Italia

MEMOCS (ISSN 2325-3444 electronic, 2326-7186 printed) is a journal of the International Research Center for the Mathematics and Mechanics of Complex Systems at the Università dell'Aquila, Italy.

Cover image: "Tangle” by $\odot$ John Horigan; produced using the Context Free program (contextfreeart.org).

PUBLISHED BY

7 mathematical sciences publishers

nonprofit scientific publishing

http://msp.org/

(C) 2017 Mathematical Sciences Publishers 
Mathematics and Mechanics of Complex Systems vol. 5 no. 3-4

Forms of the dissipation function for a class of viscoplastic 217 models

Massimo Cuomo

Tumor growth modelling by cellular automata

Ruben Interian, Reinaldo Rodríguez-Ramos, Fernando

Valdés-Ravelo, Ariel Ramírez-Torres, Celso C. Ribeiro and Aura Conci

A variational formulation for fuzzy analysis in continuum 261 mechanics

Rolf Mahnken

On the notion of stress in classical continuum mechanics

Simon R. Eugster and Christoph Glocker

MEMOCS is a journal of the International Research Center for the Mathematics and Mechanics of Complex Systems at the Università dell' Aquila, Italy.

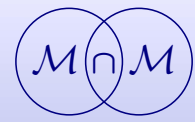

